\title{
miR-876-3p regulates glucose homeostasis and insulin sensitivity by targeting adiponectin
}

\author{
Sujith Rajan1,2, Ganesh Panzade 3,4, Ankita Srivastava1,2, Kripa Shankar', Rajesh Pandey5,*, Durgesh Kumar,2, \\ Sanchita Gupta1,2, Abhishek Gupta1, Salil Varshney1,2, Muheeb Beg1, Raj Kumar Mishra6, Ravi Shankar3,4 and \\ Anil Gaikwad1,2
}

1Division of Pharmacology, CSIR-Central Drug Research Institute, Lucknow, India

${ }^{2}$ Academy of Scientific and Innovative Research, CSIR-CDRI, Lucknow, India

3Studio of Computational Biology and Bioinformatics, Biotechnology Division, CSIR-Institute of Himalayan Bioresource Technology, Palampur, India ${ }^{4}$ Academy of Scientific and Innovative Research, CSIR-IHBT, Palampur, India

${ }^{5}$ CSIR Ayurgenomics Unit-TRISUTRA, CSIR-Institute of Genomics and Integrative Biology, New Delhi, India

${ }^{6}$ SIPS Superspeciality Hospital, Lucknow, India

Correspondence should be addressed to R Shankar or A Gaikwad: ravish@ihbt.res.in or anil_gaikwad@cdri.res.in

*(R Pandey is now at Mammalian Genetics Unit, MRC Harwell Institute, Harwell Science and Innovation Campus, Oxfordshire, UK)

\section{Abstract}

miRNA has been known to regulate diverse cellular and molecular functions. In the earlier study, we have reported that adipocytes differentiated from human mesenchymal stem cells (hMSC) on 72-h chronic insulin (CI) treatment exhibit insulin resistance (IR). Present study has further explored above model to investigate the role of early expressed miRNAs within human adipocytes to modulate differential adipokine expression as observed during IR. Our results highlight that miR-876-3p regulate glucose homeostasis and its dysregulation leads to IR. We found that miR-876-3p level is a critical determinant of adiponectin expression by virtue of its target within adiponectin 3'UTR. Regulatory effect of miR-876-3p impacts crosstalk between adiponectin and insulin signaling. Rosiglitazone treatment in Cl-induced IR adipocytes drastically reduced miR-876-3p expression and increased adiponectin level. In line with this, lentiviral-mediated inhibition of miR-876-3p expression ameliorated $\mathrm{Cl}$ and high-fat diet (HFD)-induced IR in adipocytes differentiated from hMSC and C57BL/6 mice, respectively. Our findings thus suggest that modulating miR-876-3p expression could provide novel opportunities for therapeutic intervention of obesity-associated metabolic syndrome.

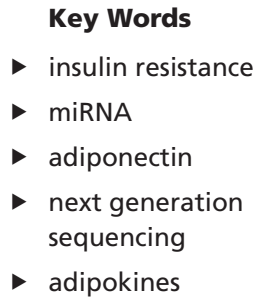

Journal of Endocrinology (2018) 239, 1-17

\section{Introduction}

miRNA and other forms of noncoding RNA have been increasingly known to play an important role in the regulation of cellular and metabolic process. According to miRbase-21, 28,645 miRNAs have been identified till November 2016. Computational prediction indicates that around $60 \%$ of human transcripts have potential miRNA binding site in $3^{\prime} \mathrm{UTR}$, making them one of the major regulators of gene expression and cellular homeostasis. The potential role of miRNA in insulin resistance (IR) and type
2 diabetes has been explored although there is potential for further investigation. Few recent studies indicate their potential as diagnostic and prognosis biomarkers (Ling et al. 2012, Chakraborty et al. 2014, Belarbi et al. 2015, Chuang et al. 2015).

Adipose tissue being the largest endocrine organ and the main site for energy storage play an important role in body glucose homeostasis. A comprehensive study toward expression profile of miRNA in adipocytes during IR 
would be important to understand the pathophysiology of IR and type 2 diabetes. Recently, several miRNAs such as miR-378 and miR-193b have been shown to regulate the expression of adiponectin (Belarbi et al. 2015, Chen et al. 2015, Gao et al. 2015, Li et al. 2016). miR-378 has been shown to regulate adiponectin level by binding to the 3'UTR of the adiponectin. Further, overexpression of miR-378 in 3T3L1 cells decreased mRNA and protein levels of adiponectin (Ishida et al. 2014). A similar study in humans have shown significant association of serum adiponectin level with increased expression of miR-193b in white adipose tissue (Belarbi et al. 2015). Few studies have implicated miRNA in degradation of adiponectin receptor. It has been shown that miR-221 regulates endothelial nitric oxide production and inflammatory response by targeting adiponectin receptor (Chen et al. 2015). These findings made us wonder what would be the effect of altered miRNA profiles in CI-induced IR adipocytes and adipokine expression.

In this study, we have identified specific adipokines, which are altered in CI-induced IR condition and are correlated with differential miRNA expression. We discovered miR-876-3p to be a regulator of adipose tissue IR by targeting adiponectin. Lentivirus-mediated overexpression and suppression studies of miR-876-3p highlighted its role in the pathophysiology of CI-induced IR. We confirmed the binding site of miR-876-3p in $3^{\prime}$ UTR of adiponectin gene using dual-luciferase reporter assays. The in vitro findings have been validated using in vivo model of HFD-induced type 2 diabetes in C57/BL6 mice. Our findings position miR-876-3p as potential target for therapeutic interventions toward the treatment of IR and type 2 diabetes.

\section{Materials and methods}

\section{hMSC culture and differentiation into white and} brown adipocytes

Human mesenchymal stem cell (hMSC) was cultured in high-glucose DMEM supplemented with 10\% fetal bovine serum (Gibco) and 1\% antibiotics. hMSC was differentiated into white as mentioned previously (Rajan et al. 2016). hMSCs were treated with differentiation cocktail containing $500 \mu \mathrm{M}$ IBMX, $5 \mu \mathrm{g} / \mathrm{mL}$ insulin, $1 \mu \mathrm{M}$ dexamethasone and $200 \mu \mathrm{M}$ indomethacin (Sigma Chemicals).

\section{IR development}

IR model was developed as described previously (Rajan et al. 2016). Briefly, differentiated adipocytes were incubated with $500 \mathrm{pM}$ of insulin (Sigma, cat no I5500) for $72 \mathrm{~h}$ wherein insulin-containing media was changed every $24 \mathrm{~h}$ to replenish the insulin level. After $72 \mathrm{~h}$, cells were washed extensively using KRH buffer $(121 \mathrm{mM}$ $\mathrm{NaCl}, 4.9 \mathrm{mM} \mathrm{KCl}, 1.2 \mathrm{mM} \mathrm{MgSO}_{4}, 0.33 \mathrm{mM} \mathrm{CaCl}$ ) supplemented with $5 \mathrm{mM}$ glucose and $0.5 \%$ BSA (step down media) prior to acute insulin.

\section{[3H] 2-deoxyglucose uptake}

Determination of 2-DOG uptake in differentiated adipocyte was performed as described previously. Nonspecific uptake was determined in the presence of cytochalasin B $(10 \mu \mathrm{M})$ during the assay which was less than $5 \%$. Glucose uptake was measured in triplicate and normalized to total protein expression as fold induction with respect to unstimulated cells. Radioactivity retained by the cell lysate was measured by liquid scintillation counter (Beckman Coulter, LS 6500, Beckman Coulter Inc.).

\section{Real-time PCR}

Total RNA was isolated from cells using TRIzol reagent (Invitrogen). First-strand cDNA synthesis was performed using high-capacity cDNA reverse transcription kit (Applied Biosystems) and subsequently used for quantitative real-time PCR analysis on Light Cycler 480 (Roche Diagnostics) using SYBR Green master mix (Roche diagnostics). Statistical analysis of the quantitative realtime PCR was done using $(2-\Delta \Delta \mathrm{Ct})$ method. Relative change in gene expression was normalized with endogenous reference gene (18S rRNA). The primer list has been provided (Supplementary material 3, see section on supplementary data given at the end of this article).

\section{Western immunoblotting}

Western blotting was performed as described previously (Rajan et al. 2016). Antibodies, pAKT Ser 473 (\#3787), pAS160 Thr 642 (\#8881), pAMPK Thr 172 (\#2535), residue were purchased from Cell Signaling Technology. Densitometric quantification of protein bands was performed using National Institute of Health ImageJ software.

\section{Adipokine array assay}

The supernatant of control and CI-induced IR adipocytes were collected after $24 \mathrm{~h}$ of $\mathrm{CI}$ treatment and adipokine 
array was performed as per manufacture protocol (R \& D Systems, Inc.).

\section{Adiponectin ELISA}

The supernatant of control and CI-induced IR adipocytes transduced with either Lenti-EV or Lenti-miR-876-3p was collected and ELISA was performed as per manufacture protocol for Duo set Adiponectin (Cat no. DY119) from R \& D Systems, Inc.

\section{Dual-Iuciferase reporter assay}

Human ADIPOQ 3'UTR, with and without miR-876-3p target binding site (Catalog No HmiT058409a-MT06 and HmiT058409b-MT06) were purchased from Genecopeia. Luciferase activity was determined using Promega dualluciferase reporter assay system (E1910) according to manufacturer protocol. Briefly, HEK293T cells were transduced with Lenti-miR-876-3p and incubated overnight. miR-876-3p overexpressing HEK293T cells were again transduced with adiponectin $3^{\prime}$ UTR with or without miR-876-3p-binding site. After 2 days, cells were washed with PBS and lysed. An equal volume of luciferase reagent was added to cell lysis and luminescence was determined using Perkin Elmer Envision 2100 luminometer. All the readings were normalized with Renilla luciferase reading.

\section{Lentivirus production and transduction}

Human pLenti-miR876-3p (overexpression of miR-876-3p, Cat.No.HmiR0505-MR03-10), pLenti-EV (control plasmid) pLenti-miR-876-3p-Inhib (inhibition of miR-876-3p, Cat. No. HmiR-AN0808-AM04) and pLenti-Con-Inhib (scramble control plasmid, Cat. No. CmiR-AN0001-AM04) were purchased from Genecopoeia (Rockville, MD, USA). Packaging plasmids (psPAX2 and pMD2.G) were gifted by Didier Trono (Addgene plasmids: 12260, 12259). Fully mature adipocytes were infected $11 \times 10^{7} / \mathrm{mL}$ of lentivirus particle at 2nd and 3rd day of CI treatment along with chronic insulin in six-well plate. The transduction was carried out with protamine sulfate at $12 \mu \mathrm{g} / \mathrm{mL}$. Further experiments were performed after $48 \mathrm{~h}$ of transduction.

\section{Mitochondrial respiration study}

Control adipocytes were transduced with either Lenti-EV or Lenti-miR-876-3p and oxygen consumption rate of (OCR) were determined using XFp Extracellular Flux analyzer (Seahorse Bioscience). Similarly, OCR was measured in CI adipocytes transduced with either LentiCon inhibitor or Lenti-miR-876-3p inhibitor. We used $1 \mu \mathrm{M}$ oligomycin, $1 \mu \mathrm{M}$ FCCP and $0.5 \mu \mathrm{M}$ Rotenone/ Antimycin A mixture as per manufacturer's protocol (Sea horse XFp Mito Stress Test kit, Cat no. 103010-100, Agilent Technologies). Readings were normalized with total protein concentration.

\section{Next generation sequencing library preparation and data analysis}

$300 \mathrm{ng}$ of RNA isolated from control and CI-induced IR adipocytes where used for next generation sequencing (NGS). For detailed procedure and analysis see the Supplementary materials and methods. The workflow of NGS data analysis is given in Supplementary Fig. 1.

\section{Animal care and treatment}

Six to eight-week-old male C57BL/6 mice were taken from animal facility of CSIR-Central Drug Research Institute. All experimental procedures were approved by the institutional animal ethics committee and were conducted in accordance with the guidelines of the Committee for the purpose of Control and Supervision of Experiments on Animals, India. Animals were acclimatized for 7 days at optimal temperature and then randomly assigned to different groups. Each group contains six animals and were kept on normal chow or HFD (60\% Kcal from fat, Cat. no. D12492 from Research diets Inc.) for 4 weeks. After 4 weeks, HFD feeding animals were randomly regrouped as either HFD+ Lenti-Con inhibitor or HFD+ miR-876-3p inhibitor. After 4 weeks of HFD feeding, mice were injected with lentiviruses at 4 - and 5-week time point through the tail vein at $1 \times 10^{7}$ plaque-forming units in $0.1 \mathrm{~mL}$ PBS. Injection of Lenti-miR-876-3p inhibitor did not affect food consumption compared to that of LentiCon inhibitor animals. Body weight and food intake were measured weekly. Animals were killed at 6 weeks after 8-h fasting. Before killing, blood was collected and then insulin $(0.75 \mathrm{U} / \mathrm{kg})$ was injected to sensitize the insulin signaling. Organs were collected and stored in liquid nitrogen.

\section{Metabolic experiments}

Intraperitoneal glucose tolerance test (IPGTT) was performed at 6 weeks after two intravenous injections of virus. For IPGTT, animals were fasted for $6 \mathrm{~h}$. Blood 
glucose was measured by Accu-Chek Active glucometer (Roche Diagnostics). For glucose tolerance test, glucose $(2 \mathrm{~g} / \mathrm{kg})$ was administered intraperitoneally and blood glucose was measured at $0,15,30,60,90$ and $120 \mathrm{~min}$ from tail tip. For intraperitoneal insulin tolerance test (ITT), mice were fasted for $5 \mathrm{~h}$ and insulin $(0.75 \mathrm{IU} / \mathrm{kg})$ was injected intraperitoneally. Blood glucose was measured at $0,15,30,60,90$ and $120 \mathrm{~min}$ from tail tip.

\section{ELISA}

Adiponectin ELISA was performed as per manufacturer's protocol (R\&D Systems, Inc.).

\section{Statistical analysis}

Results are expressed as mean \pm s.D. for at least three to five separate determinations for each experiment. Densitometry of western blot data is expressed as mean \pm S.D. of three independent experiments. Statistical significance was determined by Student's $t$-test $(P$ values $<0.05)$ and one-way ANOVA for real-time results (GraphPad Prism version 5).

\section{Results}

\section{Transcriptome analysis of control and Cl-induced IR adipocytes}

We have earlier reported that 72-h CI treatment leads to IR phenotype in adipocyte differentiated from hMSC (Rajan et al. 2016). CI treatment decreased insulinstimulated phosphorylation of AKT at Ser 473 and AS160 Thr 642 residue compared to control adipocytes (Fig. 1A). To investigate differentially expressed miRNA and mRNA at the early stage of IR, we performed NGS at $72 \mathrm{~h}$ of CI-treated adipocytes as shown in experimental design (Fig. 1B). hMSCs were isolated from five different liposuction samples and differentiated into adipocytes. CI treatment was given to all the samples and protein was isolated with/without insulin stimulation to check phosphorylation of AKT. Even though all the samples on CI treatment showed decreased phosphorylation of AKT (Ser473) and AS160 (Thr642) residue upon insulin stimulation, we randomly selected three samples for RNA-seq (Fig. 1C). The RNA sequencing data have been uploaded at CSIR-IHBT server http://scbb.ihbt.res. in:8080/ scbb/adipose.scbb/. We got 2528 upregulated and 646 downregulated expressed genes (Fig. 1D). A set of six genes (ADIPOQ - Adiponectin, C1Q And Collagen Domain Containing, LEP - leptin/obesity factor, NMB Neuromedin B, PRKAA2 - Protein Kinase AMP-Activated Catalytic Subunit Alpha 2, PPP1R3C - Protein Phosphatase 1 Regulatory Subunit 3C, PPP1R3G - Protein Phosphatase 1 Regulatory Subunit 3G), which are earlier reported in IR (Yamauchi et al. 2001, Paula et al. 2010, 2016, PazFilho et al. 2012, Zhang et al. 2014, Van Berendoncks et al. 2015, de Luis et al. 2016) appeared as potential candidates for miRNA target. This set of six genes have role in pathways for adipocytokine, AMPK signaling and insulin signaling (Table 1). The genes related to the molecular and biological process altered in CI-induced IR adipocytes are provided (Supplementary material 2 and Supplementary Fig. 2). small RNA-seq revealed 222 upregulated and 136 downregulated miRNAs (Fig. 1E). Scatter plot and heat map of differentially regulated mRNA and miRNA in control and CI-induced IR adipocytes are given in Fig. 1D and $\mathrm{E}$. The overall raw and filtered reads statistics have been available (Supplementary Tables 1 and 2).

\section{Identification of reciprocal relationships between miRNAs and targets genes}

We performed the miRNA target prediction using TAREF (Heikham \& Shankar 2010) and TargetScan (Agarwal et al. 2015); and subsequent expression negative correlation of miRNA-mRNA target. A total of 73,288 interaction pairs were obtained (PCC $\leq-0.6)$ Among these interaction pairs, 3296 interactions were found in CLASH/CLIP-seq experimental binding data. Enrichment analysis of miRNA target genes indicate glycolysis and gluconeogenesis pathway to be significantly altered (FDR <0.0004) (Table 1). The genes related to glucose homeostasis, type 2 diabetes and insulin signaling were targeted by ten miRNAs, these ten miRNA were further validated using qRT-PCR (Fig. 2A). miRNA target interaction data revealed the above ten miRNA have 426 interactions with downregulated genes, associated with pathways given (Table 1 ). Among these, four miRNAs (miR-876-3p, miR-20a-3p, miR-324-5p and miR-92a-2-5p) had targets in diabetes-related pathways (Fig. $2 \mathrm{~B}$ and D). miR-876-3p (7.01 Log2FC) and miR-92a-2-5p (7.001 Log2FC) have target within ADIPOQ ( -0.8 Log2FC) (Fig. 2C). ADIPOQ was found downregulated in CI-induced IR condition while its targeting miRNA, miR-876-3p, was upregulated (Fig. 2D). 


\begin{tabular}{l|l|l|r|}
$\begin{array}{l}\text { Journal of } \\
\text { Endocrinology }\end{array}$ & $\begin{array}{l}\text { miR-876-3p regulates adipoQ } \\
\text { expression }\end{array}$ & $\mathbf{2 3 9 : 1}$
\end{tabular}

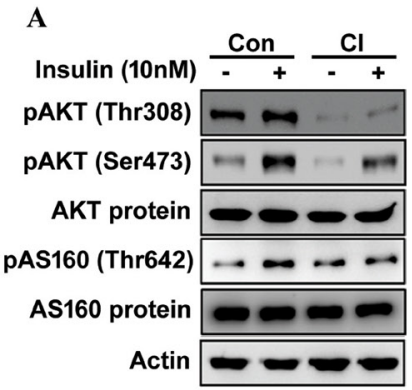

B

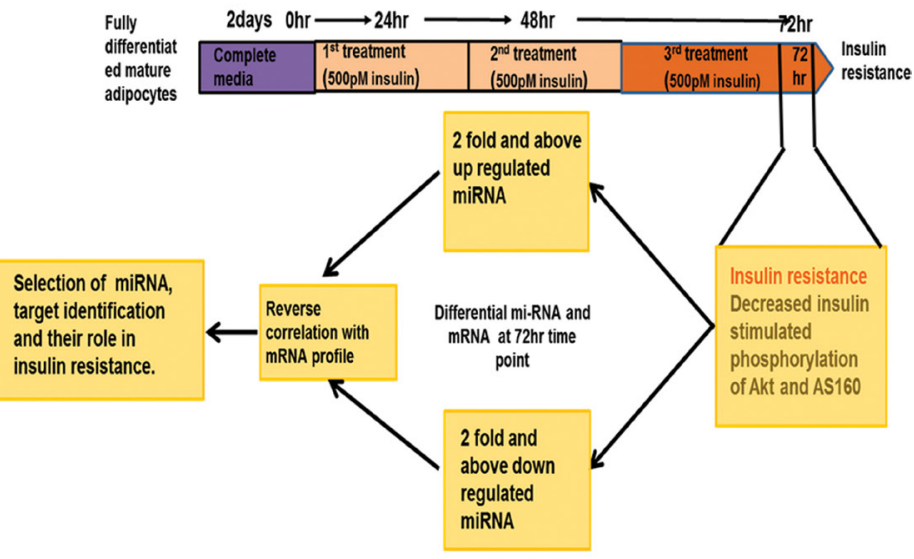

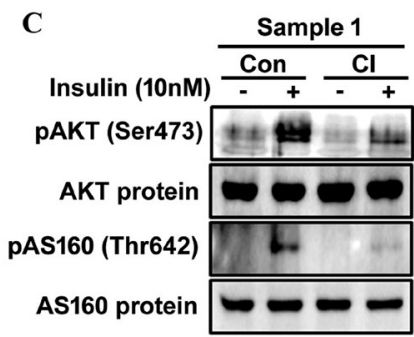

D
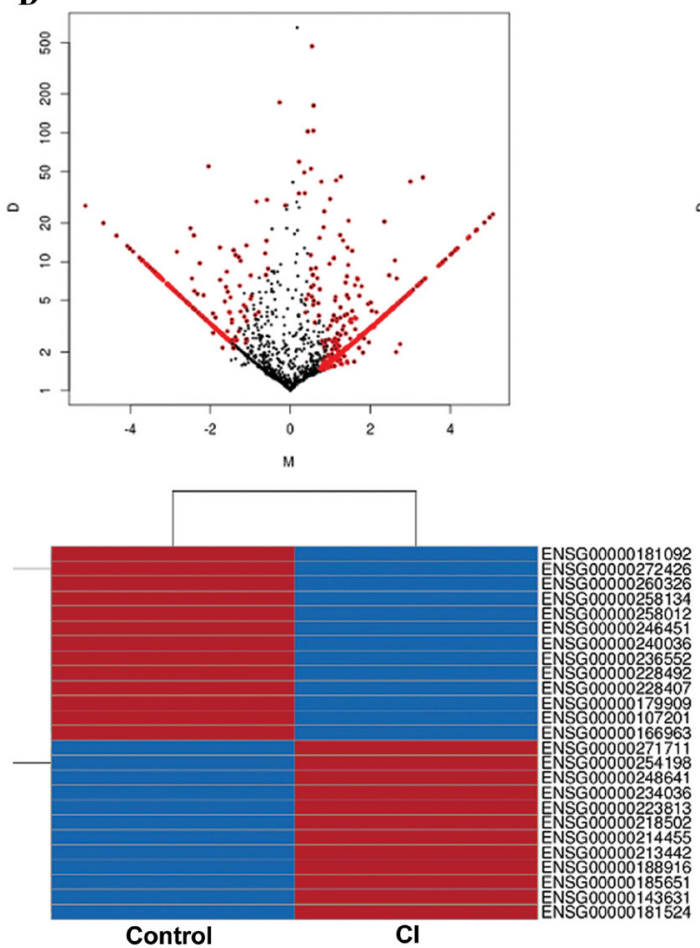

Sample 2

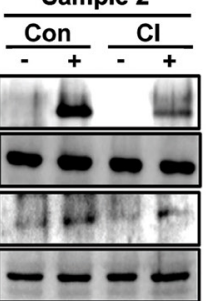

Sample 4

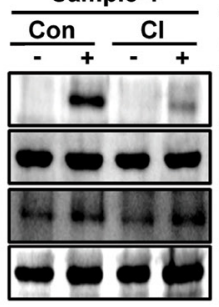
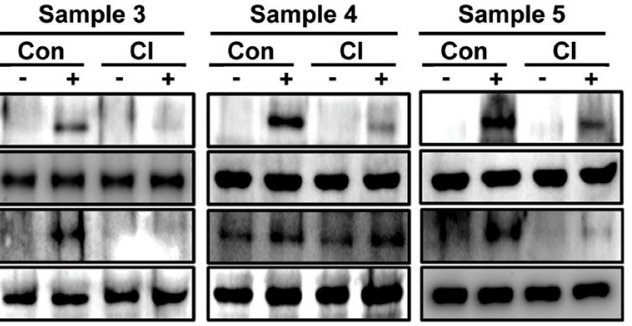

$\mathbf{E}$
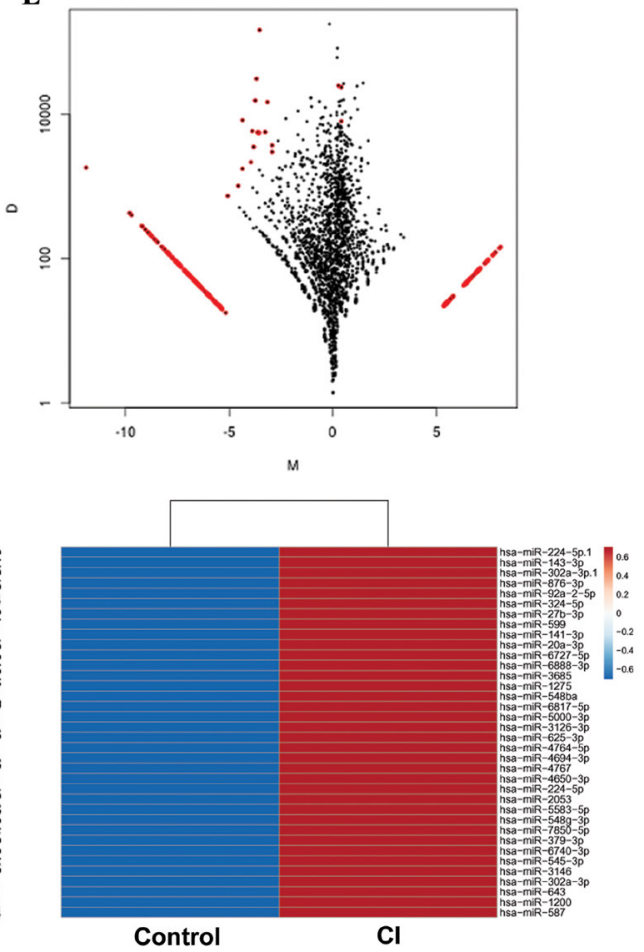

Figure 1

Transcriptome analysis of control and Cl-induced IR adipocytes. Western blot analysis of proteins involved in insulin signaling in control and $\mathrm{Cl}$-treated adipocytes, $n=3$ (A). Schematic diagram representing experimental design of differential miRNA and mRNA profiling (B). Adipocytes differentiated from hMSC (five different liposuction samples BMI $<30$ ) were given $\mathrm{Cl}$ treatment for $72 \mathrm{~h}$ and protein was isolated with/without insulin stimulation. Proteins were isolated and subjected to Western blot analysis for pAKT (Ser473), pAS160 (Thr642), AKT and AS160 protein, $n=3$. (C). MA plots and Heat map of differentially expressed mRNA and miRNA in control and Cl-induced IR adipocytes ( $\mathrm{D}$ and $\mathrm{E}$ ). A full colour version of this figure is available at https://doi. org/10.1530/JOE-17-0387. 
Table 1 Pathways of validated miRNAs target genes.

\begin{tabular}{|c|c|c|c|c|}
\hline Pathway database & Pathway name & Pathway ID & Involved genes & Adjusted $P$ value \\
\hline KEGG pathway & Adipocytokine signaling pathway & 4920 & PRKAA2, LEP, ADIPOQ & 0.000000418 \\
\hline KEGG pathway & Insulin signaling pathway & 4910 & PRKAA2, PPP1R3C & 0.0003 \\
\hline Wikipathways & AMPK signaling & WP1403 & PRKAA2, LEP, ADIPOQ & 0.000000382 \\
\hline Wikipathways & Adipogenesis & WP236 & LEP, ADIPOQ & 0.0002 \\
\hline Pathway Commons & Integration of energy metabolism & DB_ID:812 & PRKAA2, LEP & 0.0003 \\
\hline Pathway Commons & $\begin{array}{l}\text { Transcriptional regulation of white adipocyte } \\
\text { differentiation }\end{array}$ & DB_ID:57 & LEP, ADIPOQ & 0.0003 \\
\hline Pathway Commons & Developmental biology & DB_ID:11 & LEP, ADIPOQ & 0.0045 \\
\hline Pathway Commons & Metabolism & DB_ID:634 & PRKAA2, LEP & 0.0119 \\
\hline Pathway Commons & Signal transduction & DB_ID:331 & PRKAA2, NMB & 0.0203 \\
\hline
\end{tabular}

Overall pathway detailed from KEGG, Wiki and Pathway Common in database. The significant pathway having $P$ value $<0.05$ from hyper-geometric test and Bonferroni adjustment.

\section{CI-induced IR adipocytes show differential}

adipokine profiling: miR-876-3p targets adiponectin: a key insulin sensitizer

Bioinformatic analysis of differential miRNA and mRNA predicted alterations in key adipokine (adiponectin, leptin) and adipokine signaling in CI-induced IR adipocytes. To validate these, we performed an adipokine array, for which co-ordinates are given (Supplementary material 1). CI-induced IR adipocytes showed altered secretion of large number of adipokines. We found significantly decreased level of adiponectin $(P<0.0018)$ in the supernatant of CI-induced IR adipocytes. We also found that IR adipocyte produced more pro-inflammatory
A

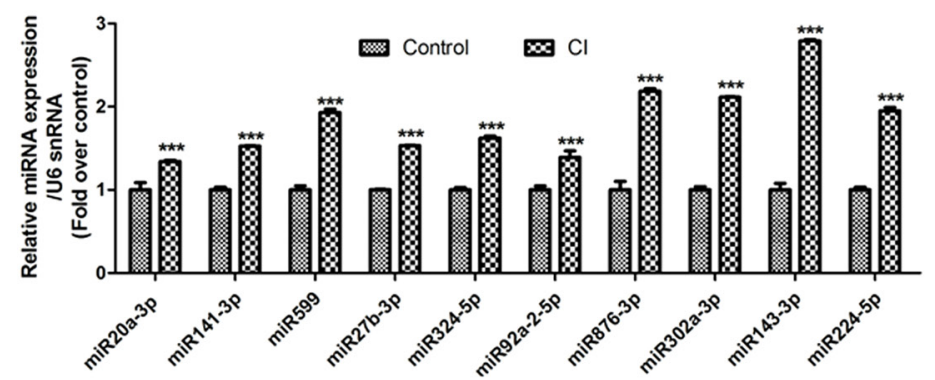

B

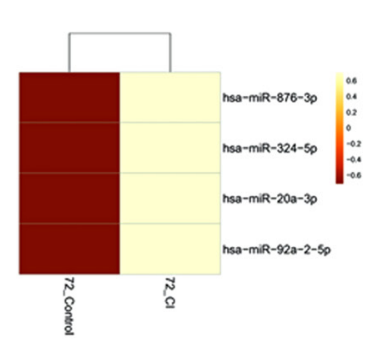

C

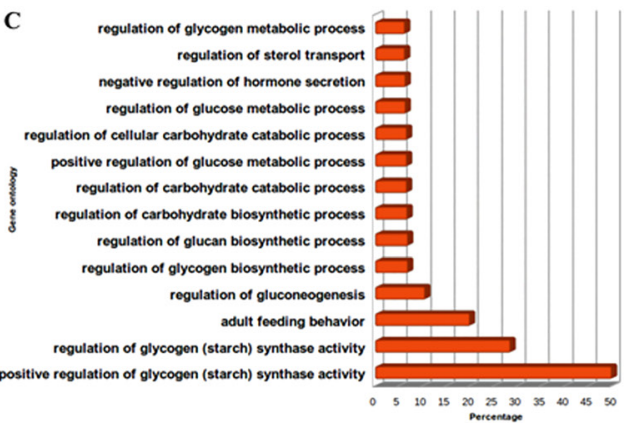

D

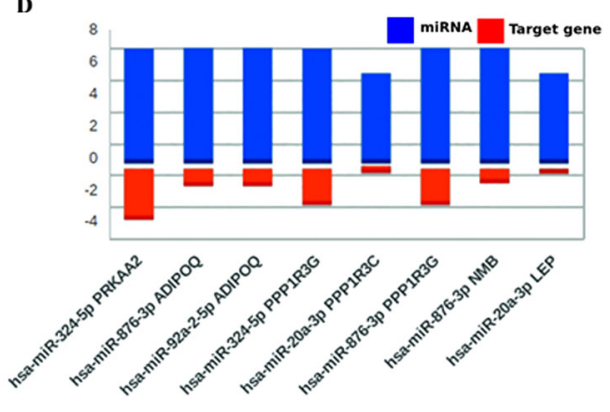

miRNA Target name

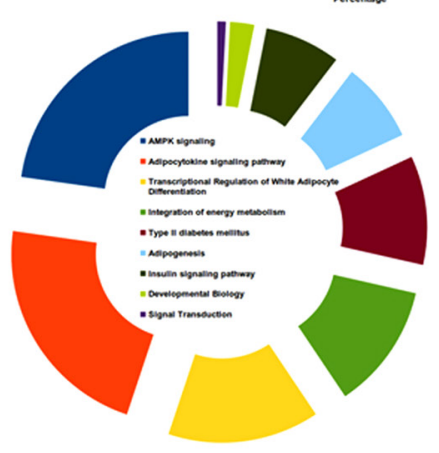

Figure 2

Identification of reciprocal relationships between miRNAs and targets genes. qPCR validation of ten differentially expressed miRNAs from control and $\mathrm{Cl}$ condition (A). Expression heatmap of ten validated miRNAs whose anti-correlation with target gene is significant (PCC <-0.6) (B). Significant pathway bar chart of miRNA target genes (C). LogFC value difference association between miRNAs and target genes. Red colored bar for target gene and blue colored bar for miRNAs (D). A full colour version of this figure is available at https://doi.org/10.1530/JOE-17-0387. 
adipokines like TNF $\alpha \quad(P<0.0026)$ and LIPOCALIN-2 $(P<0.0001)$ compared to control adipocytes. Level of adipokines, such as endocan, FGF21, RAGE, Cathepsin-L and angiopoientin-1, which are not previously reported in IR were also found to be altered in the supernatant of CI-induced IR adipocytes (Fig. 3A and B). The densitometry graphs of 58 adipokines analyzed using adipokine array are summarized (Supplementary Fig. 3A, B, C, D, E and F). Adipokine array results corroborated the findings from bioinformatic analysis of differential mRNA and miRNA expression. The long-term effect of adiponectin is mediated by PGC $1 \alpha$ and proteins involved in $\beta$-oxidation. We found decreased expression of $P G C 1 \alpha$ and CPT1A in CI-induced IR adipocytes (Fig. 3C). The bioinformatic analysis and adipokine array results suggest adiponectin to be potential target of miR-876-3p. A schematic diagram representing the interaction of miR-876-3p with 7-mer complementary nucleotide to seed sequence is given in Fig. 3D. To experimentally validate the binding, a luciferase reporter construct containing 3'UTR of human ADIPOQ with or without target site of miR-876-3p was transduced in HEK293T cells overexpressing miR-876-3p.
Relative luciferase activity was significantly $(P<0.002)$ inhibited on transduction of $A D I P O Q$ containing target sequence of miR-876-3p compared to ADIPOQ gene without seed sequence-binding site (Fig. 3E). A network view of miR-876-3p target genes is given in Fig. 4. Out of 300 target genes of miR-876-3p, more than 50\% were found interacting with each other (Supplementary material 4). Majority of which belonged to a single cluster, centered around three hub genes: MAP2K2, CAD and EP300. The clustering and network properties of miR-876-3p target genes protein-protein interactions (PPI) were performed in Cytoscape (Shannon et al. 2003). Most of these genes, including the hub genes, have been reported to be associated with adipocytes, muscles, insulin response, energy and glucose metabolism related processes.

\section{Overexpression of miR-876-3p decreased adiponectin expression and caused IR in human adipocytes}

Mature adipocytes were transduced with either Lenti-EV or Lenti-miR-876-3p (Fig. 5A). Lentivirus-mediated
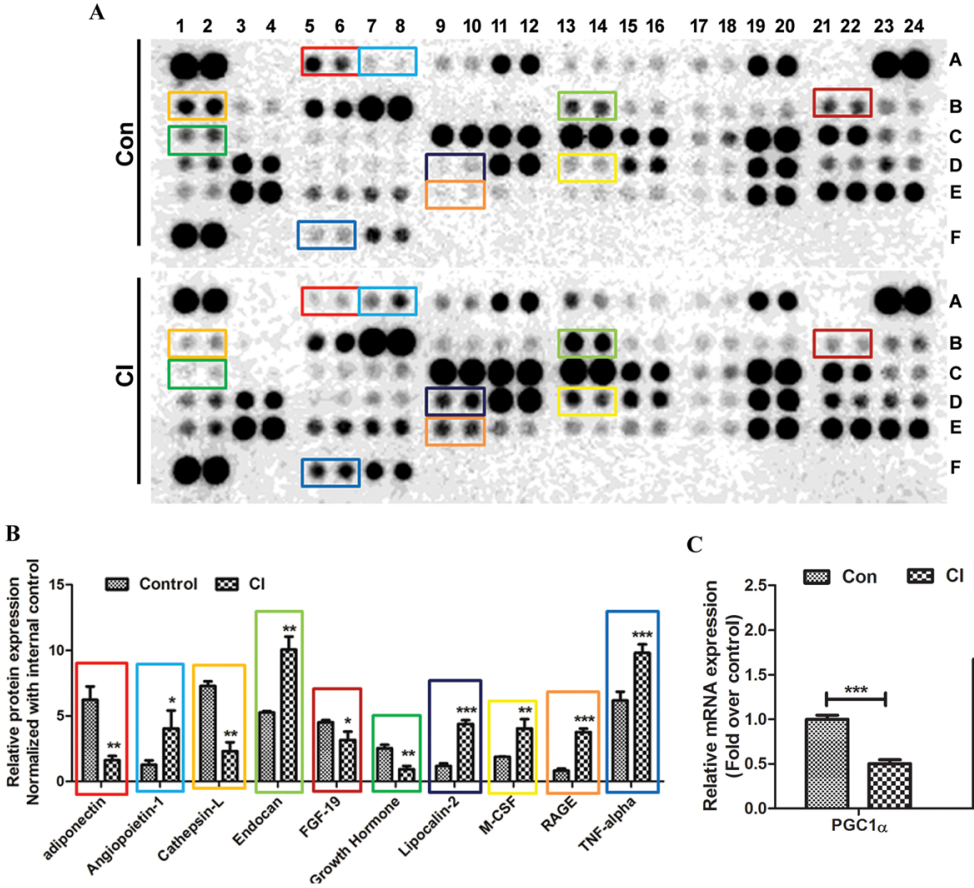

D

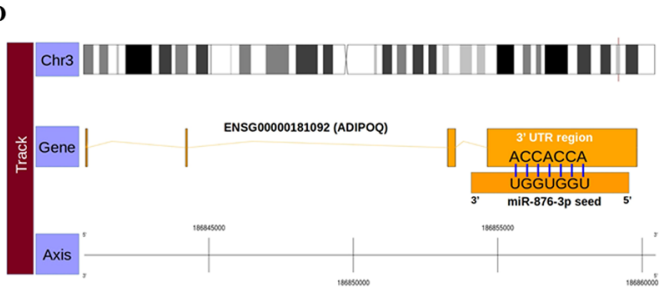

C
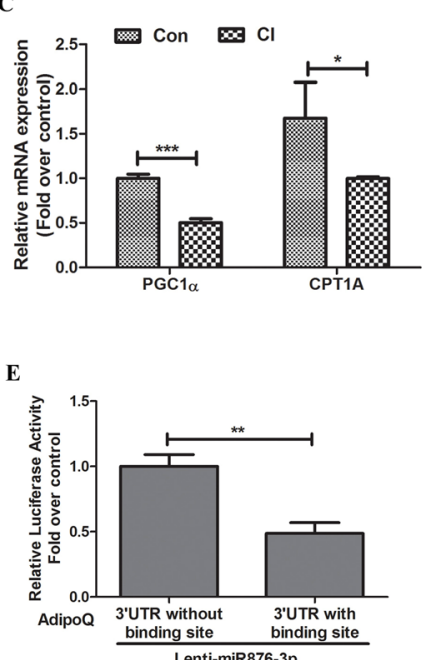

Figure 3

$\mathrm{Cl}$-induced IR adipocytes show differential adipokine profiling: miR-876-3p targets adiponectin. Supernatant of control and Cl-induced IR adipocytes were collected and different adipokines were analyzed using adipokine array kit. The dot blot of different adipokines in supernatant of control and Cl-induced IR adipocytes, $n=2$ (A). Densitometry analysis of dot blots of fig $A$, error bars represent S.D., $* P<0.05, * * P<0.01, * * * P<0.001$ as tested by Student $t$-test (B). Quantitative gene expression analysis of PGC1 $\alpha$, CPT1A in control and $\mathrm{Cl}$-induced IR adipocytes. $n=3$, error bars represent S.D., $* * P<0.01$ and $* * * P<0.001$ as tested by one-way ANOVA and Bonferroni post-test analysis (C). A case of miR-876-3p binding model to ADIPOQ in 3'UTR (D). HEK293T cells were transduced with Lenti-miR-876-3p and incubated overnight. miR-876-3p overexpressing cells were further transduced with reporter plasmid contain ADIPOQ 3' UTR with either miR-876-3p target binding site or without target binding site. After 2 days of incubation luciferase activity was measured in luminometer. Renilla luciferase activity was used for normalization and readings are presented in fold difference. $n=3$, error bars represent S.D., $* * P>0.01$ as tested by Student $t$-test (E). A full colour version of this figure is available at https://doi.org/10.1530/ JOE-17-0387. 


\begin{tabular}{|l|l|l||c|}
$\begin{array}{l}\text { Journal of } \\
\text { Endocrinology }\end{array}$ & $\begin{array}{l}\text { miR-876-3p regulates adipoQ } \\
\text { expression }\end{array}$ & $\mathbf{2 3 9 : 1}$ & $\mathbf{8}$ \\
\hline
\end{tabular}

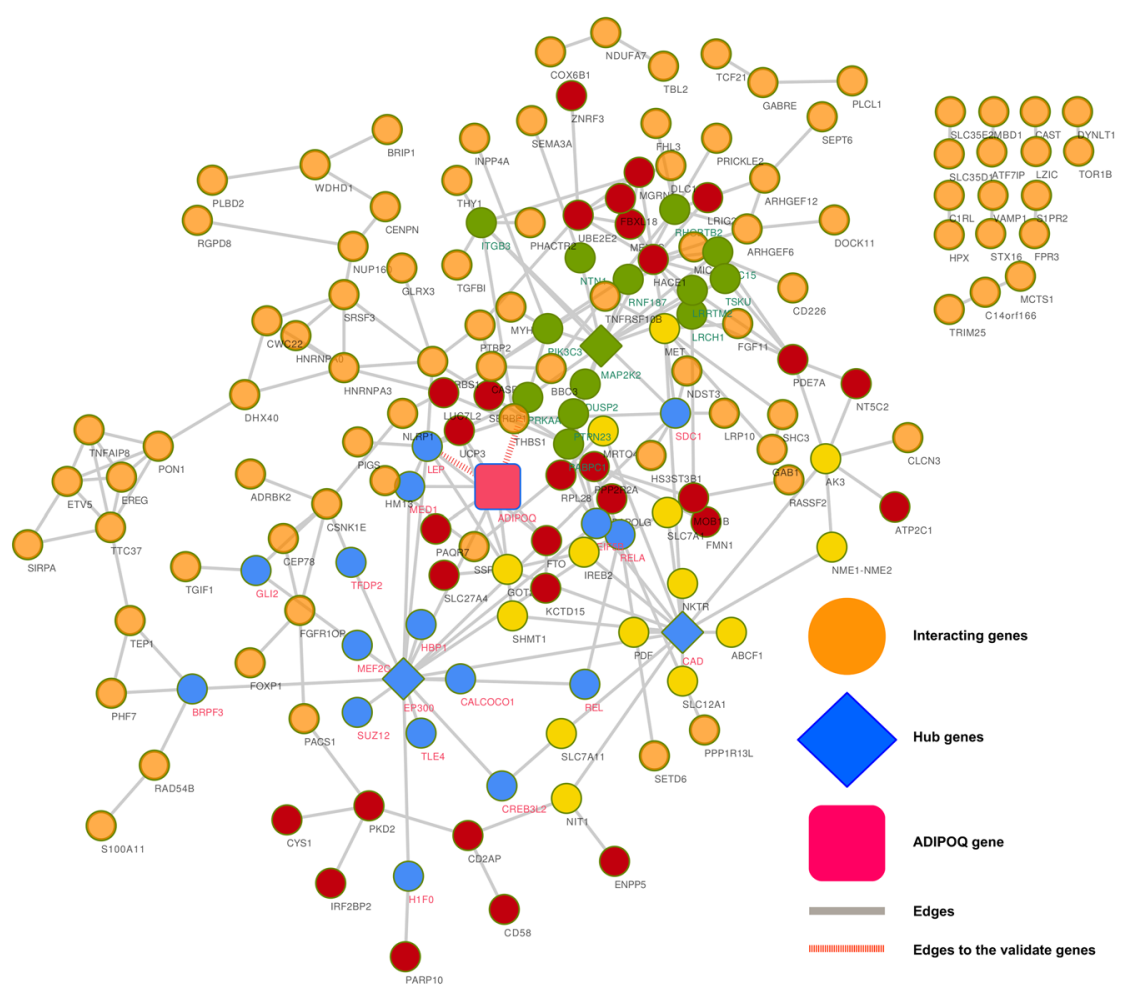

\section{Figure 4}

A network view of miR-876-3p target genes. Out of 300 target genes of miR-876-3p, more than $50 \%$ were found interacting with each other. Majority of which belonged to a single cluster centered around three hub genes: MAP2K2, CAD and EP300. Most of these genes, including the hub genes, have been reported to be associated with adipocytes, muscles, insulin response, energy and glucose metabolism related process. A full colour version of this figure is available at https:// doi.org/10.1530/JOE-17-0387. transduction of miR-876-3p in adipocytes differentiated from $\mathrm{hMSC}$ led to four- to five-fold increase in its expression (Fig. 5B). We found decreased expression of adiponectin in the background of miR-876-3p overexpression within adipocytes (Fig. 5C). miR-876-3p over-expressed adipocytes also showed increased expression of pro-inflammtaory cytokine, like $T N F \alpha$ and decreased expression of genes involved in beta-oxidation - PGC1 $\alpha$ and CPT1A (Fig. 5D), similar to CI-induced IR adipocytes (Fig. 3C) and at protein level as shown earlier (Rajan et al. 2016). The adipocytes transduced with Lenti-miR-876-3p showed significant decrease in adiponectin level in supernatant compared to Lenti-EV-transduced adipocytes (Fig. 5E). The downstream proteins in adiponectin-mediated signaling like pAMPK $(P<0.0038)$, pACC $(P<0.0033)$, pATF2 $(P<0.0067)$ and pP38 $(P<0.0005)$ were found to be less phosphorylated in Lenti-miR-876 transduced adipocytes compared to adipocytes with Lenti-EV (Fig. 5F). We checked the expression of adiponectin receptor, $A D I P O Q R 1$ and ADIPOQR2 (Target of miR876-3p), to affirm that the decrease in adiponectin signaling is not due to alteration in its receptor (Supplementary Fig. 4A). To check whether overexpression of miR-876-3p induces IR phenotype; insulin-stimulated AKT and AS160 phosphorylation was analyzed, which are nodal proteins of insulin signaling. miR-876-3p overexpressing adipocytes showed decreased phosphorylation of AKT at both the residues of Ser473 and Thr308, compared to adipocytes transduced with Lenti-EV upon insulin stimulation. We also observe similar trend in insulin-stimulated phosphorylation of AS160 in miR-876-3p overexpressing adipocytes (Fig. 5G). Further, adipocytes transduced with Lenti-miR-876-3p or Lent-EV were stimulated with $10 \mathrm{nM}$ insulin and glucose uptake was measured. We found decreased insulin-stimulated glucose uptake in adipocytes overexpressing miR-876-3p compared to control adipocytes (Lenti-EV) (Fig. 5H). The above results confirm that miR-876 attenuates insulin-stimulated phosphorylation of AKT and AS160 and thereby decreasing glucose uptake.

Inhibition of miR-876-3p in Cl-induced IR adipocytes increased adiponectin level and restored insulin signaling

CI-induced IR adipocytes were transduced with either control inhibitor or miR-876-3p inhibitor (Fig. 6A). LentimiR-876-3p inhibitor-transduced CI-induced IR adipocytes showed decreased expression of miR-876-3p compared to Lenti-con-inhibitor-transduced IR adipocytes (Fig. 6B). Relative gene expression analysis showed increased expression of adiponectin in CI-induced IR adipocytes transduced with Lenti-miR-876-3p inhibitor compared to Lenti-control inhibitor CI-induced IR adipocytes (Fig. 6C). We also found decreased expression of pro-inflammatory 
A

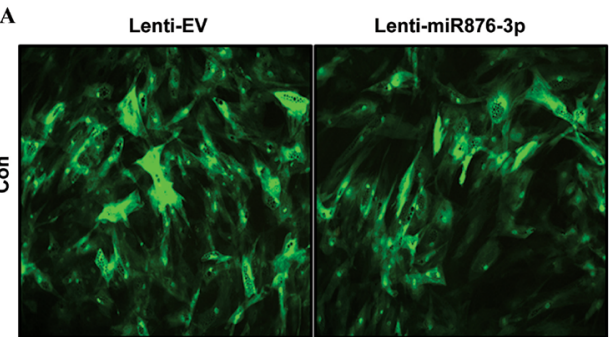

D

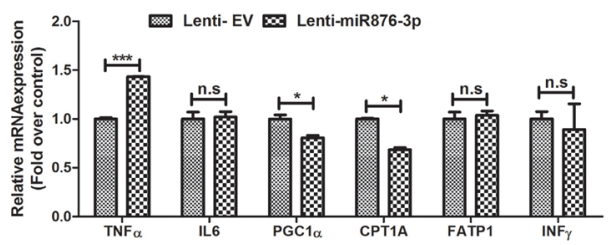

$\mathbf{F}$

G
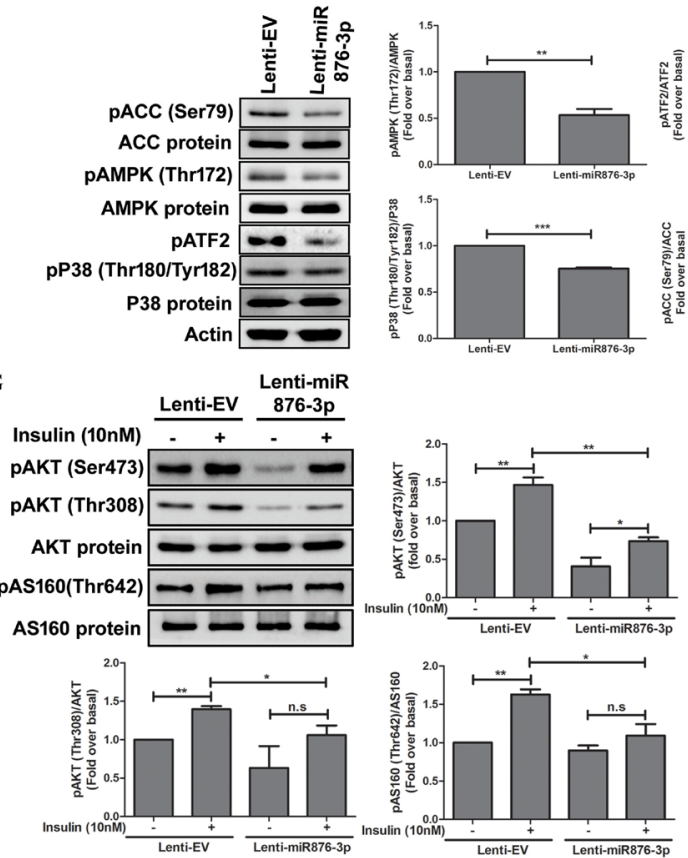

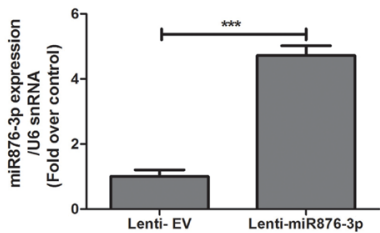

C
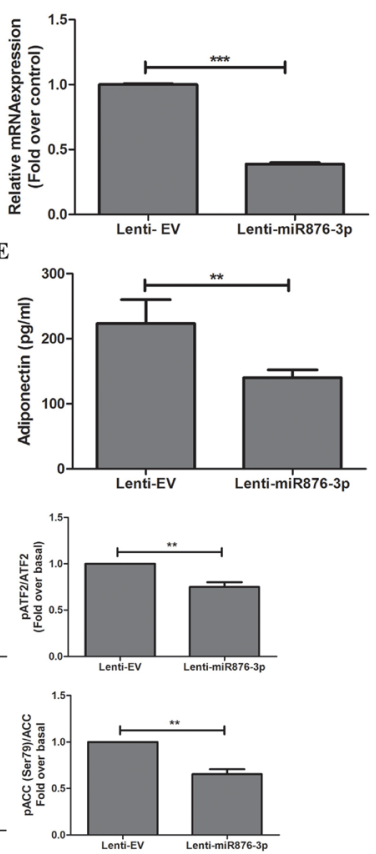

H

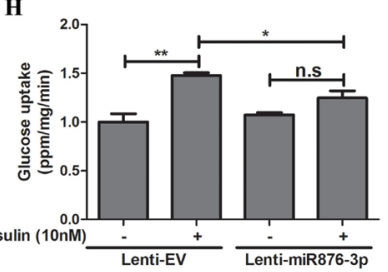

\section{Figure 5}

Overexpression of miR-876-3p decreased adiponectin expression and caused insulin resistance in human adipocytes. Adipocytes transduced with either Lenti-EV or Lenti-miR876-3p. Images were taken after $48 \mathrm{~h}$ of transduction at 10x magnification with Leica DFC 450C (A). qPCR of miR-876-3p in adipocytes transduced with either Lenti-EV or Lenti-876-3p. $n=3$, error bars represent S.D., $* * * P<0.001$ as tested by Student $t$-test (B). qPCR analysis of $A D I P O Q$ in adipocytes transduced with either Lenti-EV or Lenti-miR876-3p. RNA was isolated after $48 \mathrm{~h}$ of transduction. $n=3$, error bars represents s.D., $* * * P<0.001$ as tested by Student's $t$-test (C). qPCR analysis of genes involved in inflammation and beta-oxidation. $n=3$, error bars represent s.D., $* P<0.05$, $* * * P<0.001$ as tested by one-way ANOVA and Bonferroni post-test analysis (D). Adiponectin was measured in the supernatant of Lenti-EV or Lenti-miR-876-3p transduced adipocytes at $24 \mathrm{~h}$ interval using ELISA. The concentration of adiponectin is measured in $\mathrm{pg} / \mathrm{mL}$ of supernatant. $n=3$, error bars represent s.D. ** $P<0.01$ as tested by Student $t$-test (E). Protein isolated from adipocytes transduced with either Lenti-EV or Lenti-miR-876-3p was subjected to western blot analysis. Following antibodies were probed pACC (ser79), pAMPK (Thr172), pATF2 and pP38 (Thr180/Tyr182). The densitometry of the blots normalized with either respective protein or actin is given adjacent to the blots. $n=3$, error bars represent s.D., $* * P<0.01$ and $* * * P<0.001$ as tested by Student $t$-test (F). Fully mature adipocytes transduced with either Lenti-EV or Lenti-miR-876-3p. Protein was isolated after stimulation with insulin (10 nm) for 20 min. Isolated protein was subjected to western blot analysis and probed against pAKT (Ser473), pAKT (Thr308), pAS160 (Thr642) and their respective proteins. Densitometry analysis is shown adjacent to the blots normalized with respective proteins. $n=3$, error bars represent S.D., $* P<0.05$ and $* * P<0.01$ as tested by Student's $t$-test (G). Adipocytes transduced with either Lenti-EV or Lenti-miR-876-3p were stimulated with insulin (10 nm) for 20 min and glucose uptake was measured. Data is represented as fold difference. Glucose uptake readings were normalized with respective protein concentration. $n=3$, error bar represents s.D., * $P<0.05$ and ${ }^{*} P<0.01$ as compared by unpaired Student's $t$-test $(\mathrm{H})$. A full colour version of this figure is available at https://doi.org/10.1530/JOE-17-0387. 

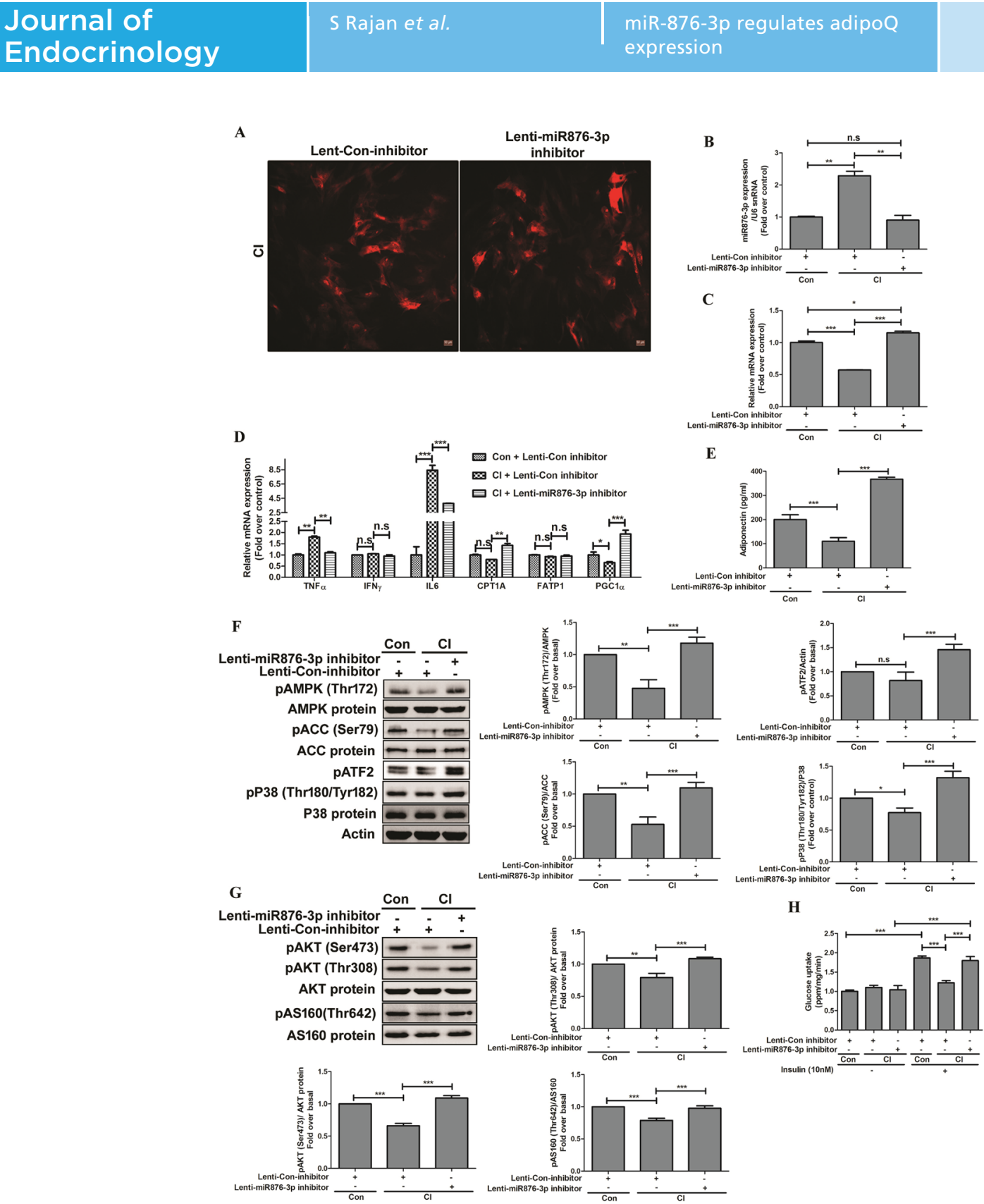

Figure 6

Inhibition of miR-876-3p in Cl-induced IR adipocytes increased adiponectin level and restored insulin signaling. Cl-treated adipocytes were either transduced with Lenti-con inhibitor or Lenti-miR876-3p inhibitor and the images were taken after $48 \mathrm{~h}$ of transduction at $10 \times$ magnification with Leica DFC 450 ( $A$ ). Quantitative gene expression analysis of miR-876-3p in control and $\mathrm{Cl}$ adipocytes transduced with either Lenti-Con inhibitor or Lenti-876-3p inhibitor. $n=3$, error bars represent S.D., $* * * P<0.001$ as tested by one-way ANOVA followed by Bonfferoni post-test analysis (B). Quantitative gene expression analysis of ADIPOQ gene in control and Cl-treated adipocytes transduced with either Lenti-Con inhibitor or Lenti-miR-876-3p inhibitor. $n=3$, error bars represents s.D., $\star * * P<0.001$ as tested by Student's $t$-test (C). qPCR analysis of genes involved in inflammation and beta-oxidation in control and Cl-treated adipocytes transduced with either Lenti-con inhibitor or Lenti-miR-876-3p inhibitor. $n=3$, error bars represent s.D., *P<0.05, $* \star \star P<0.001$ as tested by one-way ANOVA and Bonferroni post-test analysis (D). Control and Cl-treated adipocytes transduced with either Lenti-Con inhibitor or Lenti-miR-876-3p inhibitor were grown in six-well plate. Supernatant was collected at $24 \mathrm{~h}$ interval and adiponectin level was measured using ELISA. The concentration of adiponectin is measured in pg/ $\mathrm{mL}$ of supernatant. $n=3$, error bars represent S.D. ${ }^{*} P<0.01$ as tested by Student's $t$-test (E). Protein isolated from control and Cl-treated adipocytes transduced with either Lenti-Con inhibitor or Lenti-miR-876-3p inhibitor was subjected to western blot analysis. Following antibodies were probed pACC (ser79), pAMPK (Thr172), pATF2 and pP38 (Thr180/Tyr182). The densitometry of the blots normalized with either respective protein or actin is given adjacent to the blots. $n=3$, error bars represent S.D., $* * P<0.01$ and $* * * P<0.001$ as tested by Student's $t$-test (F). Control and Cl-treated adipocytes were transduced with either Lenti-Con inhibitor or Lenti-miR876-3p inhibitor. Protein was isolated after stimulation with insulin (10 nm). Isolated protein was subjected to Western blot analysis and probed against pAKT (Ser473), pAKT (Thr308), pAS160 (Thr642) and their respective proteins. Densitometry analysis is shown adjacent to the blots normalized with respective proteins. $n=3$, error bars represent S.D., ${ }^{*} P<0.05$ and ${ }^{*} P<0.01$ as tested by Student's $t$-test (G). Glucose uptake of control and $\mathrm{Cl}$-treated adipocytes transduced with either Lent-Con inhibitor or Lenti-miR-876-3p inhibitor. Insulin (10nM) stimulation was given. Data are represented as fold difference. Glucose uptake readings were normalized with respective protein concentration. $n=3$, error bar represent S.D., $* \star \star P<0.01$ as compared by one-way ANOVA and Bonferroni post-test analysis (H). A full colour version of this figure is available at https://doi.org/10.1530/JOE-17-0387. 
adipokines like $T N F \alpha$ and $I L-6$ and increased expression of PGC1 $\alpha$ and CPT1A (Fig. 6D). The above results indicate that inhibition of miR-876-3p in CI-induced IR adipocytes can restore the expression of adiponectin and reduce the expression of pro-inflammatory adipokines. We found restoration of adiponectin level in the supernatant of CI-induced IR adipocytes by inhibition of miR-876-3p (Fig. 6E). To further check whether this improves adiponectin signaling in CI-induced IR adipocytes, we analyzed the phosphorylation of AMPK, P38, ACC and ATF, involved in adiponectin signaling in CI-induced IR adipocytes transduced with either Lenti-Con inhibitor or Lenti-miR-876-3p inhibitor (Fig. 6F). The increase in adiponectin signaling was not due to increased expression of ADIPOQR1 and ADIPOQR2 in Lenti-miR-876-3p inhibitor-transduced CI-induced IR adipocytes (Supplementary Fig. 4B). To check whether the miR-876-3p inhibition in CI-induced IR adipocytes restored insulin signaling, control and CI-induced IR adipocytes were transduced with either Lenti-coninhibitor or Lenti-miR876-3p inhibitor and were subjected to western blot analysis of proteins involved in insulin signaling after stimulation with $10 \mathrm{nM}$ insulin. We found increased insulin-stimulated phosphorylation of AKT (Ser473 and Thr308) and AS160 (Thr642) in miR-876-3ptransduced CI-induced IR adipocytes compared to control inhibitor-transduced CI-induced IR adipocytes (Fig. 6G). miR-876-3p inhibited CI-induced IR adipocytes showed significant $(P<0.001)$ increase in insulin-stimulated glucose uptake compared to control inhibitor-transduced CI-induced IR adipocytes (Fig. 6H). The fold change with increase in glucose uptake was similar to non-CItreated adipocytes. The above results confirm the role of miR-876-3p in insulin signaling and maintaining insulin sensitivity via regulating adiponectin expression.

\section{miR-876-3p inhibition reverts IR by regulating the action of adiponectin}

To validate miR-876-3p regulates insulin sensitivity through adiponectin, we knocked down adiponectin in CI condition and checked phosphorylation of AKT and AS160 in the presence and absence of miR-876-3p inhibitor. We found that silencing of adiponectin in the presence of miR-876-3p inhibitor led to decreased AKT phosphorylation compared to miR-876-3p inhibitor alone treated CI-induced IR cells (Fig. 7A). One of the mechanisms by which adiponectin increases insulin sensitivity is by beta-oxidation and mitochondrial biogenesis leading to increased mitochondrial respiration (Jung \& Choi
2014). To comprehend the role of miR-876-3p in IR and adiponectin action, we studied mitochondrial respiration in adipocyte overexpressing miR-876-3p. We found, LentimiR-876-3p transduced adipocytes showed decreased basal and maximal OCR. The spare respiratory capacity was also found to be decreased in Lenti-miR-876-3p transduced adipocytes compared to Lenti-EV transduced adipocyte (Fig. 7B). As we have observed, increased adiponectin level and signaling on inhibition of miR-876-3p in CI-induced IR adipocytes; we checked whether inhibition of miR-876-3p translates to increased mitochondrial respiration in CI-induced IR adipocytes. We found inhibition of miR-876-3p in CI-induced IR adipocytes increased basal, maximal respiration and spare respiratory capacity compared to CI-induced IR adipocytes transduced with Lenti-Con inhibitor (Fig. 7C). Thiazolidinedione classes of compounds such as rosiglitazone are known for their anti-diabetic effect and are prescribed for IR treatment (Chiarelli \& Di Marzio 2008). We found miR-876-3p transduced adipocytes showed decreased adiponectin level compared to control adipocytes on rosiglitazone treatment (Fig. 7D). Supernatant of Lenti-miR-876-3p inhibitor-transduced CI-induced IR adipocytes had increased adiponectin level compared to CI-induced IR adipocytes transduced with Lenti-con-inhibitor in the presence of rosiglitazone (Fig. 7E). To check whether the increase in adiponectin level upon rosiglitazone treatment in CI-induced IR adipocytes is due to inhibition of miR-876-3p, we quantified expression of miR-876-3p in CI-induced IR adipocytes treated with rosiglitazone. We found rosiglitazone treatment led to decreased expression of miR-876-3p in CI-induced IR adipocytes (Fig. 7F). The above study recapitulates our earlier results and confirms that miR-876-3p inhibition reverts insulin signaling via increasing adiponectin level and action. More strikingly, we highlight a possible new mechanism of action by which rosiglitazone may increase adiponectin level.

\section{Inhibition of miR-876-3p leads to improved insulin} sensitivity in 4-week HFD-fed mice

The sequence alignment of mouse and human miR-876-3p precursor/mature sequence indicated $88.9 \%$ and $95.5 \%$ homology (Supplementary Fig. 5A and B). Importantly, the mouse AdipoQ 3'UTR contained the miR-876-3p-binding site as well (Supplementary Fig. 5B). As four-week HFDfed mice show hyperinsulinemia and are reported to have decreased level of adiponectin (Fraulob et al. 2010, Liu et al. 2013), we checked the expression of miR-876-3p in eWAT of 4-week HFD-fed mice (Fig. 8A). Similar to earlier 


\begin{tabular}{|l|l|l||c|}
$\begin{array}{l}\text { Journal of } \\
\text { Endocrinology }\end{array}$ & $\begin{array}{l}\text { miR-876-3p regulates adipoQ } \\
\text { expression }\end{array}$ & $\mathbf{2 3 9 : 1}$ & $\mathbf{1 2}$ \\
\hline
\end{tabular}
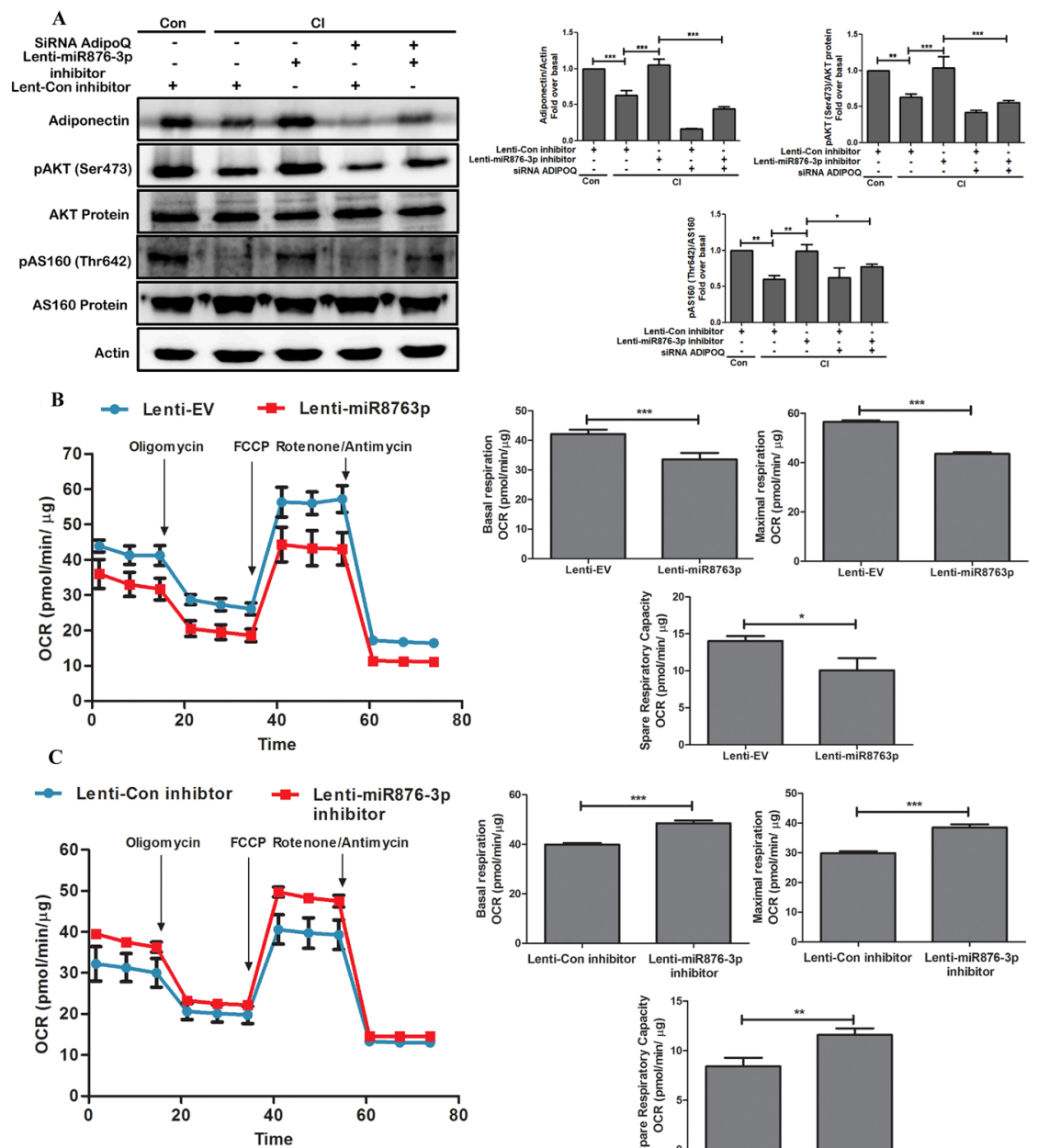

D
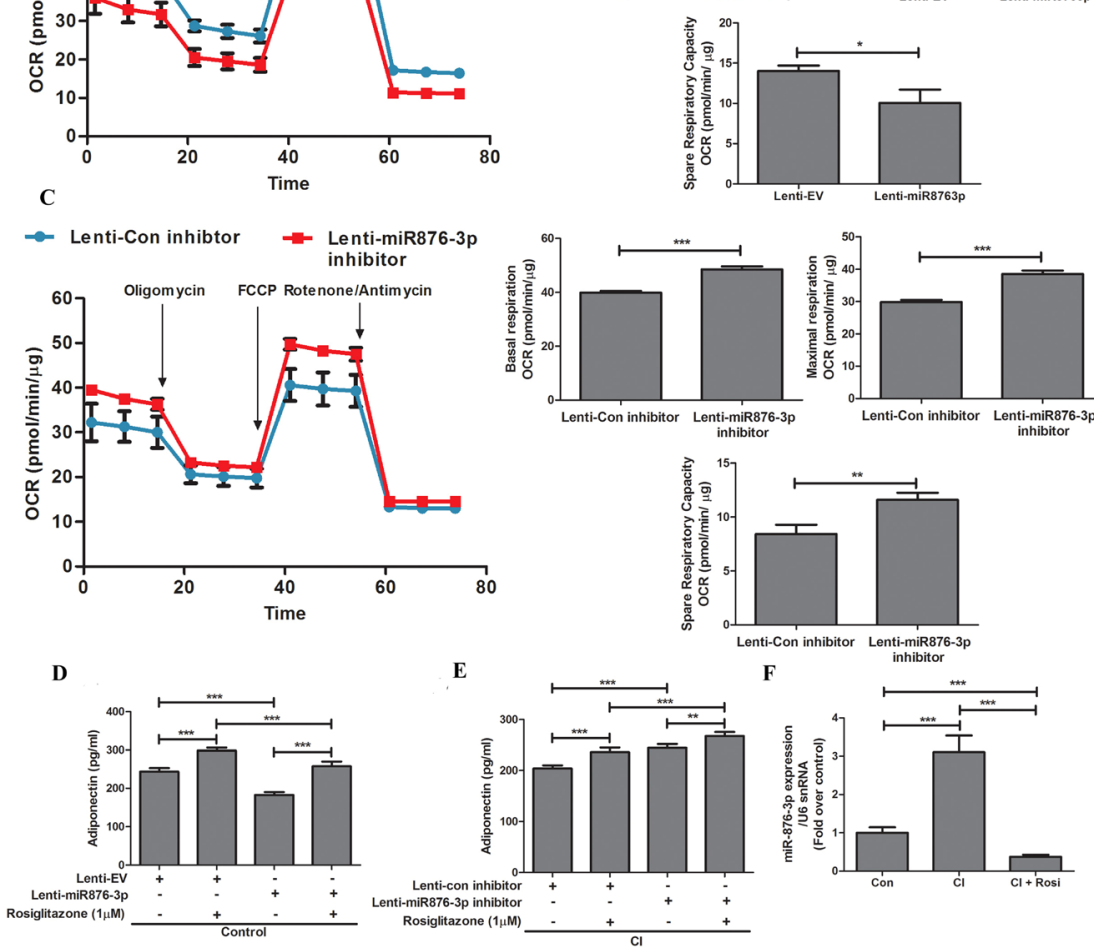

\section{Figure 7}

miR-876-3p inhibition reverts insulin resistance by regulating the action of adiponectin. siRNA-mediated adiponectin knockdown Cl-induced IR adipocytes were transfected with either Lenti-Con inhibitor or Lenti-miR-876-3p inhibitor and $10 \mathrm{nM}$ insulin pulsing was given for 20 min. Isolated protein was subjected to Western blot analysis and probed against adiponectin, pAKT (Ser473), pAS160 (Thr642) and their respective proteins. Densitometry analysis is shown adjacent to the blots normalized with respective proteins or actin. $n=3$, error bars represent s.D., $* P<0.05$ and $* \star P<0.01$ as compared by one-way ANOVA and Bonferroni post-test analysis (A). Mitochondrial respiration study of adipocyte transduced with either Lenti-EV or Lenti-miR-876-3p. OCR at basal level and in the presence of ATP synthase inhibitor ( $1 \mu \mathrm{M}$ oligomycin), proton uncoupler ( $1 \mu \mathrm{M}$ FCCP) and electron transport chain inhibitors $(0.5 \mu \mathrm{M}$ Rotenone/Antimycin mix) was measured with Seahorse Bioscience XFp Extracellular Flux analyzer. $n=3$, s.D. represent standard deviation, $* * P<0.01$ and $* * * P<0.001$ as tested by Student $t$-test $(\mathrm{B})$. Mitochondrial respiration study of $\mathrm{Cl}$-induced IR adipocytes transduced with either Lenti-Con inhibitor or Lenti-miR-876-3p inhibitor. OCR was measured similar to above experiment. $n=3$, s.D. represent standard deviation, $* * P<0.01$ and $* * * P<0.001$ as tested by Student $t$-test (C). Adiponectin level was measured in supernatant of control adipocytes transduced with either Lenti-EV or Lenti-miR-876-3p with or without $24 \mathrm{~h}$ rosiglitazone treatment. $n=3$, error bars represent S.D., $* * * P<0.001$ as tested by one-way ANOVA Bonferroni post-test analysis (D). Adiponectin level was measured in supernatant of Cl-induced IR adipocytes transduced with either Lenti-Con inhibitor or Lenti-miR-876-3p inhibitor. Rosiglitazone treatment was given as shown in figure for $24 \mathrm{~h}$. $n=3$, error bars represent s.D., $* \star P<0.01, * \star \star P<0.001$ as tested by one-way ANOVA Bonferroni post-test analysis (E). Quantitative gene expression analysis of miR-876-3p in con and Cl-treated adipocytes after $24 \mathrm{~h}$ rosiglitazone $(1 \mu \mathrm{M})$ as shown in figure. $n=3$, error bars represent s.D., $* * * P<0.001$ as tested by one-way ANOVA and Bonferroni post-test analysis (F). A full colour version of this figure is available at https://doi.org/10.1530/JOE-17-0387. 
reports, we found decreased $A D I P O Q$ expression level in eWAT and serum of HFD-fed mice compared to chow-fed mice (Fig. 8B and C). Next, we checked whether inhibition of miR-876-3p in HFD mice improves adiponectin level and thereby insulin sensitivity. We intravenously injected Lenti-miR-876-3p inhibitor to HFD mice as shown in schematic diagram (Fig. 8D), which resulted in significant decrease in miR-876-3p expression in eWAT (Fig. 8E). We found increased expression and level of adiponectin in eWAT and serum of Lenti-miR-876-3p inhibitor-injected HFD mice (Fig. 8F and G). The restoration of adiponectin expression also led to increased expression of $P G C 1 \alpha$ and CPT1A (Supplementary Fig. 5D). Body weight and eWAT weight of mice injected with Lenti-miR876-3p were found to be decreased compared to Lenti-Con inhibitor-injected HFD mice (Fig. $8 \mathrm{H}$ and I). To further elucidate the effect of miR-876-3p inhibition on insulin sensitivity, we performed IPGTT and ITT. Remarkably, we found decreased fasting glucose and improved glucose tolerance along with enhanced insulin sensitivity in LentimiR-876-3p-inhibited HFD mice (Fig. 8J, K, L, M and N). To further investigate the molecular basis of increased glucose tolerance and insulin sensitivity in Lenti-miR-876-3pinhibited mice, we analyzed insulin-stimulated signaling. Lenti-miR-876-3p inhibition resulted in increased insulinstimulated phosphorylation of AKT and AS160 in eWAT of HFD mice. We also found increased phosphorylation of ACC, AMPK and P38 key proteins involved in adiponectin signaling in Lenti-miR-876-3p inhibitor-injected HFD mice compared to Lenti-Con inhibitor-injected HFD mice (Fig. 8O), which was not due to increase in $A D I P O Q R 1$ and $A D I P O Q R 2$ expression (Supplementary Fig. 5C) Taken together, in vivo results confirm that miR-876-3p affects glucose homeostasis and insulin signaling by regulating adiponectin.

\section{Discussion}

In this study, we have demonstrated that miR-876-3p regulates glucose homeostasis and its dysregulation leads to obesity-associated metabolic syndrome. Since no study specific to human adipocytes IR were performed earlier with respect to early expression of miRNA and their role in differential adipokine expression during IR, our study highlights the role of miRNA in IR and those which are responsible for differential adipokine profile in IR adipocytes. Further, we found that the miR-876-3p is a critical determinant of adiponectin expression by virtue of target within adiponectin 3'UTR. These effects of
miR-876-3p could be greatly consolidated and augmented by crosstalk between adiponectin and insulin signaling. In line with this idea, Lenti viral-mediated inhibition of miR-876-3p expression ameliorated CI and HFD-induced IR in adipocytes differentiated from hMSC and C57BL/6 mice, respectively. Our findings thus suggest that modulating miR-876-3p expression could provide novel opportunities for therapeutic intervention during obesityassociated metabolic syndrome.

The target genes of each miRNA based on bioinformatics target prediction were categorized according to their function and were integrated to larger protein network. Wiki-pathway, KEGG, Pathway Commons and David functional annotation tool was used for the above mentioned purpose and to identify the biological process in which majority of targets were involved.

Overexpression of miR-876-3p in human adipocytes resulted in attenuation of insulin signaling and adiponectin level with concomitant increases in the expression proinflammatory cytokines such as TNF $\alpha$ and lipocalin. It was demonstrated that increased level of miR-876-3p in response to EV71 infection leads to decreased cAMP response element-binding protein (CREB5) expression, which in turn affects PI3-AKT signaling (Wang et al. 2016b). This substantiates our finding that increased expression of miR-876-3p in CI-induced IR adipocytes is one of the determinants resulting in decreased insulin-stimulated PI3-AKT pathway. The above study also corroborates well with our finding that miR-876-3p inhibition leads to decreased expression of pro-inflammatory cytokines in CI-induced IR adipocytes suggesting the role of latter in regulation of inflammatory micromilieu. miR876 has been found to be elevated in type 1 diabetes patient (Satake et al. 2018). It has also been showed that miR876-5p is elevated in human trophoblast during gestational diabetes (Bari et al. 2016). However, from these studies, it will be premature to conclude the role of miR-876 in diabetes. Presently, it would be too early to conclude whether miR876 induces IR or vice versa. The present study establishes the role of miR876 and its targets in IR pathophysiology. Since we have checked transcriptome level changes at early stage of IR phenotype development, it will be appropriate to state these changes are cause and not consequence of IR.

The incumbency of Lenti-miR-876-3p inhibitor to revert insulin resistance in adiponectin silenced CI-induced IR adipocytes further validates the action of miR-876-3p and prominence of adiponectin in maintaining insulin sensitivity. The insulin-sensitizing effect of adiponectin is 

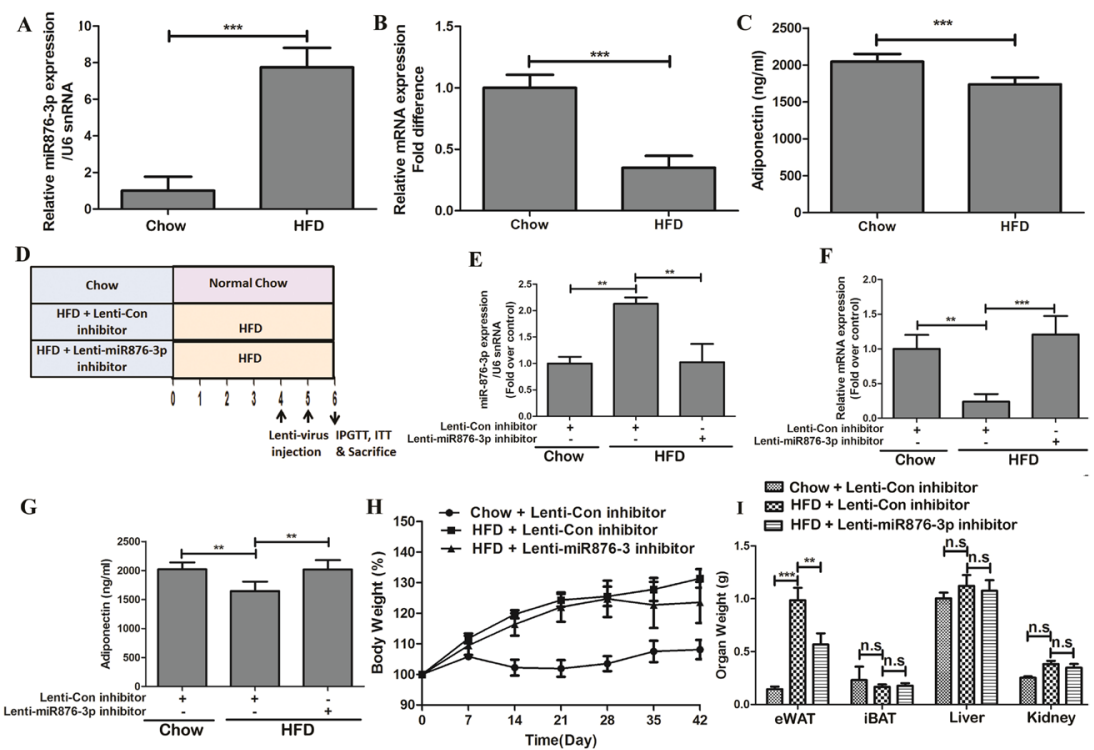

Chow + Lenti-Con inhibitor HFD + Lenti-Con inhibitor
GFD + Lenti-miR876-3p inhibito
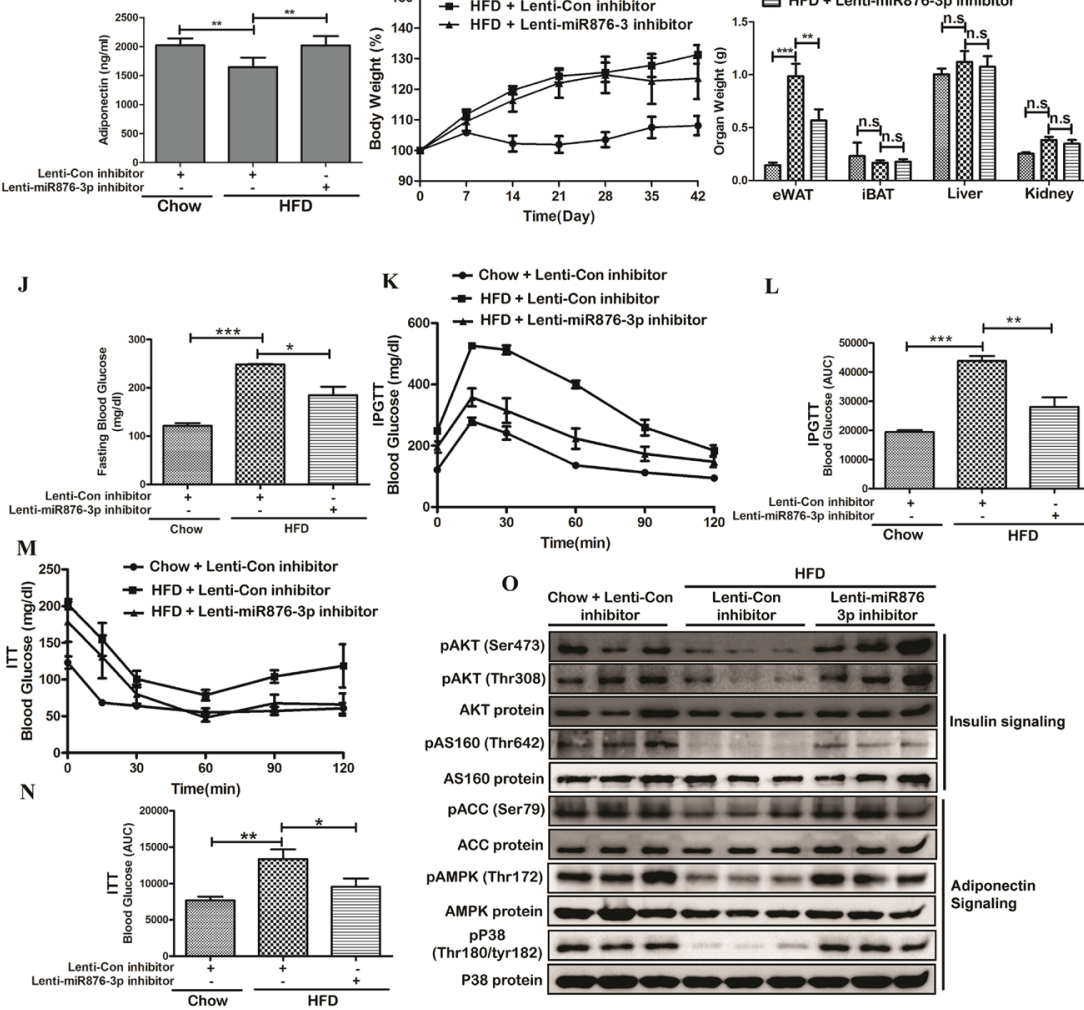

Figure 8

Inhibition of miR-876-3p lead to improved insulin sensitivity in 4-week HFD-fed mice. Quantitative gene expression analysis of miR-876-3p in eWAT of chow and HFD mice, $n=6$, error bars represent S.D., $* * * P<0.001$ as tested by Student's $t$-test (A). QPCR analysis of ADIPOQ in eWAT of chow and HFD mice. $n=6$, error bars represent s.D., $* * * P<0.001$ as tested by Student's $t$-test (B). Serum adiponectin level of chow and HFD mice represented in $n g / m L$. $n=6$, error bars represent S.E.M., $* * * P<0.001$ as tested by Student's $t$-test (C). Schematic diagram of representing Lenti-miR-876 inhibition experiment in 4-week HFD-fed mice (D). Quantitative gene expression analysis of miR-876-3p in eWAT of chow and HFD-fed mice injected with either Lenti-con inhibitor or Lenti-miR-876-3p inhibitor. $n=5$, error bars represent s.D., $* * P<0.01$ as tested by one-way ANOVA and Bonferroni post-test analysis (E). Quantitative gene expression analysis of adipoQ in eWAT of chow and HFD-fed mice injected with either Lenti-con inhibitor or Lenti-miR-876-3p inhibitor, $n=5$, error bars represent S.D. ${ }^{*} P<0.01$ and $* * * P<0.001$ as tested by one-way ANOVA followed by Bonferroni post-test analysis (F). Serum adiponectin level of chow and HFD-fed mice injected with either Lenti-con inhibitor or Lenti-miR-876-3p inhibitor. $n=5$, error bars represent s.E.M., $* * P<0.01$ as tested by one-way ANOVA followed by Bonferroni post-test analysis (G). Body weight in percentage of chow and HFD-fed mice injected with either Lenti-con inhibitor or Lent-miR-876-3p inhibitor. $n=5(\mathrm{H})$. Weight of eWAT, iBAT, liver, kidney of chow and HFD-fed mice injected with Lenti-con inhibitor or Lenti-miR-876-3p inhibitor (I). Fasting blood glucose level of chow and HFD-fed mice injected with either Lenti-con inhibitor or Lenti-miR-876-3p inhibitor, $n=5$, error bars represent s.E.M., ${ }^{*} P<0.05$ and $* * * P<0.001$ as tested by one-way ANOVA followed by Bonferroni post-test analysis (J). IPGTT and its area under curve (AUC) of chow and HFD-fed mice injected with either Lenti-con inhibitor or Lenti-876-3p inhibitor. $n=5$, error bars represent S.E.M., $* * P<0.01$ and $* * * P<0.001$ as tested by one-way ANOVA and Bonferroni post-test analysis (K and L). ITT and it AUC of chow and HFD-fed mice injected with either Lenti-con inhibitor or Lenti-miR-876-3p inhibitor. $n=5$, error bars represent s.E.M., ${ }^{*} P<0.01$ and $* * P<0.001$ as tested by one-way ANOVA and followed by Bonferroni post-test analysis ( $M$ and N). Protein isolated after insulin pulsing from eWAT of chow and HFD-fed mice injected with either Lenti-con inhibitor or Lenti-miR-876-3p inhibitor were subjected to western blot analysis. Following proteins were probed to check insulin and adiponectin signaling as shown in figure, $n=3(\mathrm{O})$. A full colour version of this figure is available at https://doi.org/10.1530/JOE-17-0387. 
derived by its increased beta-oxidation and mitochondrial biogenesis activity leading to accelerated mitochondrial respiration (Jung \& Choi 2014). One of the studies suggests low serum level of adiponectin and blunted stimulatory effect of adiponectin on activation of AMPK $\alpha 1$ in metabolic syndrome patients (Van Berendoncks et al. 2015). The decreased mitochondrial respiration observed in LentimiR-876-3p-transduced adipocytes can be correlated to the decreased adiponectin expression and signaling. Further, the decreased adiponectin expression on treatment with rosiglitazone in Lenti-miR-876-3p-transduced adipocytes compared to control adipocytes reconfirms the inhibitory effect of miR-876-3p on adiponectin. Rosiglitazonemediated increase in adiponectin level and signaling in CI-induced IR adipocytes was further potentiated by inhibition of miR-876-3p.

Adiponectin is known to inhibit the effect of TNFa and pro-inflammatory cytokines (Mandal et al. 2010). Increased expression of TNF $\alpha$ observed in CI-induced IR adipocytes supernatant can be correlated to the decreased expression of adiponectin in miR-876-3p over-expressed adipocytes. Hence, it is plausible that miR-876-3p favors pro-inflammatory environment by targeting adiponectin. Adipose tissue inflammation has been considered to be the leading cause of IR and metabolic syndrome (Shoelson et al. 2006). As we have identified the expression of miR-876-3p in human adipocytes at early stage of insulin resistance, it corroborates with decreased adiponectin expression and increased levels of pro-inflammatory cytokines observed in CI-induced IR adipocytes. Literature evidence suggests TNF $\alpha$ to be a negative regulator of adiponectin (Lim et al. 2008). Adiponectin has been reported for its anti-inflammatory activity (Dalamaga \& Christodoulatos 2015, Ehsan et al. 2016, Wang et al. 2016a), but from current study, it is difficult to establish whether the decreased level of adiponectin led to increased expression of proinflammatory cytokines or vice versa. As TNF $\alpha$ has been reported to suppress the expression of adiponectin (Kim et al. 2005, He et al. 2016), it may be pertinent to conclude that former may have causal role in deciding the micromilieu of adipose tissue and causing IR.

Interestingly, expression of miR-876-3p in eWAT of HFD-fed obese mice was consistently increased and adiponectin serum and gene expression were markedly decreased. We further demonstrated that miR-876-3p regulates insulin signaling and glucose metabolism. Notably, Lenti viral-mediated miR-876-3p inhibition in mice increased adiponectin level and abolishes
HFD-induced gain in body weight and eWAT. In addition to that, silencing of miR-876 also decreased HFD-induced hyperglycemia and improved glucose tolerance and insulin sensitivity. Since, we found significantly decreased expression of miR-876-3p in liver and adipocyte, but not in muscle of mice injected with miR-876-3p inhibitor (Supplementary Fig. 5E and F), it would be fascinating to study the role of miR-876-3p in liver and its contribution in reverting IR. These results suggest that increased miR-876-3p expression may be a driving factor of obesity-associated metabolic abnormalities. Taken together, suppression of adipose miR-876 is sufficient to prevent features of obesityassociated metabolic syndrome.

Observing the critical impact of miR-876-3p, a further exploration of other significant target genes was done to evaluate therapeutic aspect of miR-876-3p. It was found that miR-876-3p targets 300 genes in the system with majority of them associated with obesity and diabetic/IR phenotype. A thorough network analysis was performed to get the functional map of these genes. Surprisingly, $>50 \%$ of these genes interacted with each other in PPI network. More than $20 \%$ of these interacting genes belonged to a single cluster centered on MAP2K2, CAD and EP3OO (hub genes), which are well acknowledged for obesity-related IR. Even adiponectin also belonged to this same cluster. MAP2K2 dysregulation is attributed in obesity-induced diabetes and IR. CAD is associated to MAPK system as well as maintains glucose homeostasis in liver. In adipocytes, $C A D$ along with $X B P 1$ act against obesity and during obesity-induced diabetes its expression is reported to be compromised (Reardon \& Weber 1987, Deng et al. 2018). Another hub gene of miR-876 target system was EP-300, a highly important cofactor, which has been reported to increase the expression of GLUT-2 gene. Its down regulation is associated with IR and diabetes, and it is considered as an important gene for beta cell proliferation (Ban et al. 2002, Wong et al. 2018). In general, these 300 genes displayed functional association with relevant processes like leptin signaling, muscle cells, glucose metabolism, energy metabolism and insulin signaling. Figure 4 provides the overall network view of this critical cluster, controlled by miR-876-3p. Further details on miR-876 targets have been provided (Supplementary material 4). Therefore, miR-876-3p stands as a very promising therapeutic target, which appears controlling mainly a large number of genes working in a modular fashion toward IR phenotype. 


\section{Conclusion}

In summary, our study demonstrates the importance of adipose miR-876-3p in glucose metabolism and insulin signaling through regulation of adiponectin. It is more desirable to check the effect of miR-876-3p in adiponectinknockout mice to validate the phenotypic change brought by miR-876-3p, but the technical difficulties in acquiring adiponectin-knockout mice render it beyond scope of this manuscript. Downregulating miR-876-3p in obese mice prevents obesity-induced metabolic damage. As we have performed all the studies in adipocyte differentiated from hMSC and in IR model close to human physiology, our finding has greater implications in the development of the therapeutic targets for type- 2 diabetes.

\section{Supplementary data}

This is linked to the online version of the paper at https://doi.org/10.1530/ JOE-17-0387.

\section{Declaration of interest}

The authors declare that there is no conflict of interest that could be perceived as prejudicing the impartiality of the research reported.

\section{Funding}

This research work is supported by Department of Biotechnology and Department of Science and Technology DBT government of India project GAP0179 (CSIR-CDRI) and GAP0188 (CSIR-IHBT). Research work is also partly supported by CSIR-CDRI Network project: Toward holistic understanding of complex diseases: Unraveling the threads of complex disease (THUNDER) Project No: BSC0102 (CSIR-CDRI).

\section{Author contribution statement}

Sujith Rajan wrote the manuscript and did most of the in vitro studies. Ganesh Pazande wrote manuscript and did bioinformatic analysis of next generation sequencing data. Ankita Srivastava prepared lentivirus particles for the gene modulation studies. Kripa Shankar performed in vivo studies and checked the manuscript. Dr Rajesh Pandey performed next generation sequencing and corrected the manuscript. Abhishek Gupta and Sanchita Gupta performed SiRNA experiments. Durgesh Kumar and Salil Varshney were involved in animal experiments. Dr Muheeb Beg optimized initial experiments. Dr Raj Kumar Mishra performed liposuction surgery and provided samples. Dr Ravi Shankar and Dr Anil N Gaikwad conceptualized the idea and corrected the manuscript.

\section{Acknowledgments}

S R, A G and K S are supported by SRF-UGC; D K and A S are supported by SRF CSIR, New Delhi; G P is supported by SRF (DST-INSPIRE). S G is supported by DBT-JRF. S V is supported by ICMR-SRF. The authors acknowledge Prof. Nuzhat Husain (Ex Director), Dr Ram Manohar Lohia Institute of Medical Science for approval from stem cell ethics committee. They appreciate help and support provided by staff of Sushrut Institute of Plastic Surgery Hospital for collection of liposuction sample. This manuscript bears CSIRCDRI communication number: 9720 .

\section{References}

Agarwal V, Bell GW, Nam JW \& Bartel DP 2015 Predicting effective microRNA target sites in mammalian mRNAs. eLife $\mathbf{4}$ e05005. (https:// doi.org/10.7554/eLife.05005)

Ban N, Yamada Y, Someya Y, Miyawaki K, Ihara Y, Hosokawa M, Toyokuni S, Tsuda K \& Seino Y 2002 Hepatocyte nuclear factor1alpha recruits the transcriptional co-activator p300 on the GLUT2 gene promoter. Diabetes 51 1409-1418. (https://doi.org/10.2337/ diabetes.51.5.1409)

Bari MF, Ngo S, Bastie CC, Sheppard AM \& Vatish M 2016 Gestational diabetic transcriptomic profiling of microdissected human trophoblast. Journal of Endocrinology 229 47-59. (https://doi. org/10.1530/JOE-15-0424)

Belarbi Y, Mejhert N, Lorente-Cebrián S, Dahlman I, Arner P, Rydén M \& Kulyté A 2015 MicroRNA-193b controls adiponectin production in human white adipose tissue. Journal of Clinical Endocrinology and Metabolism 100 E1084-E1088.

Chakraborty C, Doss CG, Bandyopadhyay S \& Agoramoorthy G 2014 Influence of miRNA in insulin signaling pathway and insulin resistance: micro-molecules with a major role in type- 2 diabetes. Wiley Interdisciplinary Reviews: RNA 5 697-712. (https://doi. org/10.1002/wrna.1240)

Chen CF, Huang J, Li H, Zhang C, Huang X, Tong G \& Xu YZ 2015 MicroRNA-221 regulates endothelial nitric oxide production and inflammatory response by targeting adiponectin receptor 1. Gene 565 246-251. (https://doi.org/10.1016/j.gene.2015.04.014)

Chiarelli F \& Di Marzio D 2008 Peroxisome proliferator-activated receptor-gamma agonists and diabetes: current evidence and future perspectives. Vascular Health and Risk Management 4 297-304. (https:// doi.org/10.2147/VHRM.S993)

Chuang TY, Wu HL, Chen CC, Gamboa GM, Layman LC, Diamond MP, Azziz R \& Chen YH 2015 MicroRNA-223 expression is upregulated in insulin resistant human adipose tissue. Journal of Diabetes Research 2015943659.

Dalamaga M \& Christodoulatos GS 2015 Adiponectin as a biomarker linking obesity and adiposopathy to hematologic malignancies. Hormone Molecular Biology and Clinical Investigation 23 5-20.

de Luis DA, Izaola O, de la Fuente B, Primo D, Fernandez Ovalle H \& Romero E 2016 rs1501299 polymorphism in the adiponectin gene and their association with total adiponectin levels, insulin resistance and metabolic syndrome in obese subjects. Annals of Nutrition and Metabolism 69 226-231. (https://doi.org/10.1159/000453401)

Deng Y, Wang ZV, Gordillo R, Zhu Y, Ali A, Zhang C, Wang X, Shao M, Zhang Z, Iyengar P, et al. 2018 Adipocyte Xbp1s overexpression drives uridine production and reduces obesity. Molecular Metabolism 11 1-17. (https://doi.org/10.1016/j.molmet.2018.02.013)

Ehsan M, Singh KK, Lovren F, Pan Y, Quan A, Mantella LE, Sandhu P, Teoh H, Al-Omran M \& Verma S 2016 Adiponectin limits monocytic microparticle-induced endothelial activation by modulation of the AMPK, Akt and NFkappaB signaling pathways. Atherosclerosis 245 1-11. (https://doi.org/10.1016/j.atherosclerosis.2015.11.024)

Fraulob JC, Ogg-Diamantino R, Fernandes-Santos C, Aguila MB \& Mandarim-de-Lacerda CA 2010 A mouse model of metabolic syndrome: insulin resistance, fatty liver and non-alcoholic fatty pancreas disease (NAFPD) in C57BL/6 mice fed a high fat diet. Journal of Clinical Biochemistry and Nutrition 46 212-223. (https://doi. org/10.3164/jcbn.09-83)

Gao Q, Yao X \& Zheng J 2015 MiR-323 inhibits prostate cancer vascularization through adiponectin receptor. Cellular Physiology and Biochemistry 36 1491-1498. (https://doi.org/10.1159/000430313) 
He Y, Lu L, Wei X, Jin D, Qian T, Yu A, Sun J, Cui J \& Yang Z 2016 The multimerization and secretion of adiponectin are regulated by TNFalpha. Endocrine 51 456-468. (https://doi.org/10.1007/s12020-0150741-4)

Heikham R \& Shankar R 2010 Flanking region sequence information to refine microRNA target predictions. Journal of Bioscience 35 105-118. (https://doi.org/10.1007/s12038-010-0013-7)

Ishida M, Shimabukuro M, Yagi S, Nishimoto S, Kozuka C, Fukuda D, Soeki T, Masuzaki H, Tsutsui M \& Sata M 2014 MicroRNA-378 regulates adiponectin expression in adipose tissue: a new plausible mechanism. PLoS ONE 9 e111537. (https://doi.org/10.1371/journal. pone.0111537)

Jung UJ \& Choi MS 2014 Obesity and its metabolic complications: the role of adipokines and the relationship between obesity, inflammation, insulin resistance, dyslipidemia and nonalcoholic fatty liver disease. International Journal of Molecular Sciences 15 6184-6223. (https://doi.org/10.3390/ijms15046184)

Kim KY, Kim JK, Jeon JH, Yoon SR, Choi I \& Yang Y 2005 c-Jun $\mathrm{N}$-terminal kinase is involved in the suppression of adiponectin expression by TNF-alpha in 3T3-L1 adipocytes. Biochemical and Biophysical Research Communications 327 460-467. (https://doi. org/10.1016/j.bbrc.2004.12.026)

Li Y, Cai X, Guan Y, Wang L, Wang S, Fu Y, Gao X \& Su G 2016 Adiponectin upregulates MiR-133a in cardiac hypertrophy through AMPK activation and reduced ERK1/2 phosphorylation. PLOS ONE 11 e0148482. (https://doi.org/10.1371/journal.pone.0148482)

Lim JY, Kim WH \& Park SI 2008 GO6976 prevents TNF-alpha-induced suppression of adiponectin expression in 3T3-L1 adipocytes: putative involvement of protein kinase C. FEBS Letters 582 3473-3478. (https://doi.org/10.1016/j.febslet.2008.09.012)

Ling HY, Hu B, Hu XB, Zhong J, Feng SD, Qin L, Liu G, Wen GB \& Liao DF 2012 MiRNA-21 reverses high glucose and high insulin induced insulin resistance in 3T3-L1 adipocytes through targeting phosphatase and tensin homologue. Experimental and Clinical Endocrinology and Diabetes 120 553-559. (https://doi. org/10.1055/s-0032-1311644)

Liu Y, Turdi S, Park T, Morris NJ, Deshaies Y, Xu A \& Sweeney G 2013 Adiponectin corrects high-fat diet-induced disturbances in muscle metabolomic profile and whole-body glucose homeostasis. Diabetes 62 743-752. (https://doi.org/10.2337/db12-0687)

Mandal P, Park PH, McMullen MR, Pratt BT \& Nagy LE 2010 The anti-inflammatory effects of adiponectin are mediated via a heme oxygenase-1-dependent pathway in rat Kupffer cells. Hepatology $\mathbf{5 1}$ 1420-1429. (https://doi.org/10.1002/hep.23427)

Paula GS, Souza LL, Cabanelas A, Bloise FF, Mello-Coelho V, Wada E, Ortiga-Carvalho TM, Oliveira KJ \& Pazos-Moura CC 2010 Female mice target deleted for the neuromedin B receptor have partial resistance to diet-induced obesity. Journal of Physiology 588 1635-1645. (https:// doi.org/10.1113/jphysiol.2009.185322)

Paula GS, Souza LL, Bressane NO, Maravalhas R, Wilieman M, BentoBernardes T, Silva KR, Mendonca LS, Oliveira KJ \& Pazos-Moura CC 2016 Mice with deletion of neuromedin B receptor exhibit decreased oral glucose-stimulated insulin release. Hormone and Metabolic Research 48 854-861. (https://doi.org/10.1055/s-0042-115643)

Paz-Filho G, Mastronardi C, Wong ML \& Licinio J 2012 Leptin therapy, insulin sensitivity, and glucose homeostasis. Indian Journal of Endocrinology and Metabolism 16 S549-S555. (https://doi. org/10.4103/2230-8210.105571)

Rajan S, Shankar K, Beg M, Varshney S, Gupta A, Srivastava A, Kumar D, Mishra RK, Hussain Z, Gayen JR, et al. 2016 Chronic hyperinsulinemia reduces insulin sensitivity and metabolic functions of brown adipocyte. Journal of Endocrinology 230 275-290. (https:// doi.org/10.1530/JOE-16-0099)

Reardon MA \& Weber G 1987 Regulation by insulin of liver carbamoylphosphate synthase II (glutamine-hydrolysing). Biochemistry Journal 244 345-350. (https://doi.org/10.1042/bj2440345)

Satake E, Pezzolesi MG, Md Dom ZI, Smiles AM, Niewczas MA \& Krolewski AS 2018 Circulating miRNA profiles associated with hyperglycemia in patients with type 1 diabetes. Diabetes $\mathbf{6 7}$ 1013-1023. (https://doi.org/10.2337/db17-1207)

Shannon P, Markiel A, Ozier O, Baliga NS, Wang JT, Ramage D, Amin N, Schwikowski B \& Ideker T 2003 Cytoscape: a software environment for integrated models of biomolecular interaction networks. Genome Research 13 2498-2504. (https://doi.org/10.1101/gr.1239303)

Shoelson SE, Lee J \& Goldfine AB 2006 Inflammation and insulin resistance. Journal of Clinical Investigation 116 1793-1801. (https://doi. org/10.1172/JCI29069)

Van Berendoncks AM, Stensvold D, Garnier A, Fortin D, Sente T, Vrints CJ, Arild SS, Ventura-Clapier R, Wisloff U \& Conraads VM 2015 Disturbed adiponectin - AMPK system in skeletal muscle of patients with metabolic syndrome. European Journal of Preventive Cardiology 22 203-205. (https://doi.org/10.1177/2047487313508034)

Wang H, Zhang H, Zhang Z, Huang B, Cheng X, Wang D, la Gahu Z, Xue Z, Da Y, Li D, et al. 2016a Adiponectin-derived active peptide ADP355 exerts anti-inflammatory and anti-fibrotic activities in thioacetamide-induced liver injury. Scientific Reports 6 19445. (https:// doi.org/10.1038/srep19445)

Wang RY, Weng KF, Huang YC \& Chen CJ $2016 b$ Elevated expression of circulating miR876-5p is a specific response to severe EV71 infections. Scientific Reports 6 24149. (https://doi.org/10.1038/srep24149)

Wong CK, Wade-Vallance AK, Luciani DS, Brindle PK, Lynn FC \& Gibson WT 2018 The p300 and CBP transcriptional coactivators are required for beta-cell and alpha-cell proliferation. Diabetes 67 412-422. (https://doi.org/10.2337/db17-0237)

Yamauchi T, Kamon J, Waki H, Terauchi Y, Kubota N, Hara K, Mori Y, Ide T, Murakami K, Tsuboyama-Kasaoka N, et al. 2001 The fat-derived hormone adiponectin reverses insulin resistance associated with both lipoatrophy and obesity. Nature Medicine 7 941-946. (https://doi. org/10.1038/90984)

Zhang Y, Xu D, Huang H, Chen S, Wang L, Zhu L, Jiang X, Ruan X, Luo X, Cao P, et al. 2014 Regulation of glucose homeostasis and lipid metabolism by PPP1R3G-mediated hepatic glycogenesis. Molecular Endocrinology 28 116-126. (https://doi.org/10.1210/ me.2013-1268)

Received in final form 14 June 2018

Accepted 2 July 2018

Accepted Preprint published online 2 July 2018 http://joe.endocrinology-journals.org

https://doi.org/10.1530/JOE-17-0387
() 2018 Society for Endocrinology Published by Bioscientifica Ltd. Printed in Great Britain 